Copyright (C) 2006 IEEE. Reprinted from

IEEE Transactions on Geoscience and Remote Sensing, 2006; 44

(8):2041-2054

This material is posted here with permission of the IEEE. Such permission of the IEEE does not in any way imply IEEE endorsement of any of the University of Adelaide's products or services. Internal or personal use of this material is permitted. However, permission to reprint/republish this material for advertising or promotional purposes or for creating new collective works for resale or redistribution must be obtained from the IEEE by writing to pubs-permissions@ieee.org.

By choosing to view this document, you agree to all provisions of the copyright laws protecting it. 


\title{
Detecting Scene Changes Using Synthetic Aperture Radar Interferometry
}

\author{
Mark Preiss, Member, IEEE, Douglas A. Gray, Member, IEEE, and Nick J. S. Stacy, Member, IEEE
}

\begin{abstract}
In repeat-pass interferometric synthetic aperture radar (SAR), man-made scene disturbances are commonly detected by identifying changes in the mean backscatter power of the scene or by identifying regions of low coherence. Change statistics such as the sample mean backscatter-power ratio and the sample coherence, however, are susceptible to high false-alarm rates unless the change in the mean backscatter power is large or there is sufficient contrast in scene coherence between the changed and unchanged regions of the image pair. Furthermore, as the sample mean backscatter-power ratio and sample coherence measure different properties of a SAR image pair, both change statistics need to be considered to properly characterize scene changes. In this paper, models describing the changed and unchanged regions of a scene are postulated, and the detection problem is expressed in a Bayesian hypothesis-testing framework. Forming the loglikelihood ratio gives a single sufficient statistic, encoding changes in both the coherence and the mean backscatter power, for discriminating between the unchanged- and changed-scene models. The theoretical detection performance of the change statistic is derived and shows a significant improvement over both the sample mean backscatter-power ratio and sample coherence change statistics. Finally, the superior detection performance of the loglikelihood change statistic is demonstrated using experimental data collected using the Defence Science and Technology Organisation's Ingara X-band airborne SAR.
\end{abstract}

Index Terms-Change detection, clairvoyant detector, coherence, hypothesis testing, log-likelihood ratio, synthetic aperture radar $(\mathrm{SAR})$ interferometry.

\section{INTRODUCTION}

$\mathbf{S}$ YNTHETIC aperture radar (SAR) is a coherent imaging technique capable of generating fine-resolution images of the complex radar backscatter of a scene from large ranges. An important application of SAR is the detection of temporal changes in a scene. Change detection is an application to which SAR is particularly well suited as SARs can consistently produce high-quality well-calibrated imagery with good geolocation accuracy.

Two forms of change detection in repeat-pass SAR imagery may be considered, namely coherent and incoherent change detection. Incoherent change detection identifies changes in the mean backscatter power of the scene by comparing sample estimates of the mean backscatter power taken from the repeatpass image pair. Typically, the sample estimates are obtained

Manuscript received September 5, 2005; revised December 19, 2005.

M. Preiss and N. J. S. Stacy are with the Defence Science and Technology Organisation, Edinburgh 1500, Australia (e-mail: mark.preiss@dsto.defence. gov.au).

D. A. Gray is with the Cooperative Research Centre for Sensor Signal and Information Processing and with the University of Adelaide, Adelaide, SA 5005, Australia.

Digital Object Identifier 10.1109/TGRS.2006.872910 by spatially averaging the image pixel intensities (amplitude squared) over local regions in the image pair. The mean backscatter power of a scene is determined by the structural and dielectric properties of the scene, and thus may be used to detect changes in soil or vegetation moisture content or surface roughness. Coherent change detection, on the other hand, uses the magnitude of the sample complex cross correlation of an interferometric SAR image pair to quantify changes in the observed amplitude and phase of the image pixels. Since the observed pixel amplitude and phase is sensitive to the relative spatial geometry of the scattering contributions within a pixel, coherent change detection has the potential to detect very subtle scene changes. The observed pixel amplitude and phase, however, are also highly sensitive to mismatch in the acquisition apertures and aberrations in the image-formation processing [1]. Coherent change detection thus requires additional interferometric processing steps to mitigate these sources of image decorrelation. The mean backscatter power and complex correlation coefficient measure different properties of a scene and, therefore, both coherent and incoherent change statistics should be considered to properly characterize scene changes [2].

The sample mean backscatter-power ratio and the magnitude of the sample cross-correlation coefficient, commonly referred to as the sample coherence, have been employed in the literature to detect a variety of different types of scene change as well as classify different target types. A number of papers [3], [4] have demonstrated the ability to discriminate between different crops and vegetation types using the sample mean backscatter power and coherence, and classifiers based on these statistics have been proposed [5], [6]. The scene coherence has also been used to identify areas inundated by flood [7], and its use in monitoring urban development has been examined [8]. The sensitivity of the scene coherence in detecting subtle scene changes has been demonstrated in [9], in which low-coherence tracks, possibly due to scene disturbances arising from grazing sheep, were detected. In [10], the interferometric processing of 1-m resolution airborne X-band SAR imagery was used to identify changes in an earthworks site.

In this paper, the detection and localization of fine-scale man-made scene changes such as the passage of vehicles or personnel across an open field are considered. The small spatial scale of such scene changes and the desire to localize the changes using fine-resolution imagery and small estimation windows can result in poor detection performance using the sample mean backscatter-power ratio or sample coherence. Furthermore, while the mean backscatter and sample coherence provide complementary information about the scene changes, fusing the detections from the change statistics is problematic as, pixelwise, the detections not do necessarily agree. In this paper, models describing the changed and unchanged regions 
of a scene are postulated and the detection problem is expressed in a Bayesian hypothesis-testing framework [9]. This leads to the formulation of a new log-likelihood change statistic that encodes both coherence and mean backscatter power changes for detecting scene changes. Through theoretical analysis, simulation, and application to experimental data, it is shown that the new change statistic yields superior detection performance compared to that of either the mean backscatter-power ratio or the sample coherence.

In Section II, an image model for a repeat-pass interferometric image pair is discussed, leading to the formulation of the changed- and unchanged-scene hypotheses detailed in Section III. The performance of the sample mean backscatterpower ratio and sample coherence change statistics is analyzed and compared in Sections IV and V. In Sections VI-VIII, the new log-likelihood change statistic is developed and the theoretical performance of the associated clairvoyant detector [11] is derived. Section IX evaluates the performance degradation that may be expected for a practical generalized likelihood-ratio change statistic in which the unknown parameters are replaced with their maximum-likelihood estimates (MLEs). Finally, in Section X, the change statistics are applied to experimental repeat-pass SAR imagery acquired with the Defence Science and Technology Organisation (DSTO)'s Ingara X-band SAR and the improved detection performance is demonstrated.

\section{INTERFEROMETRIC SAR IMAGE MODEL}

For homogeneous natural distributed target environments, the scene is commonly modeled as consisting of a large number of discrete scatterers having a uniform random phase and identically distributed random amplitude [12], [13]. The nature of the observed complex radar backscatter $f$ has been studied in the literature [14], and may be modeled as a zero-mean circular complex Gaussian random variable, with variance $\sigma_{f}^{2}$ being the mean backscatter power. The mean backscatter power $\sigma_{f}^{2}$ is a geophysical quantity describing the local interaction of the propagating incident electromagnetic wave with the scene's scattering volume. It is thus determined by the structural and electromagnetic properties of the scattering elements in the volume, such as their size, shape, density, dielectric constant, as well as the properties of the incident wave such as the wavelength, polarization, and incidence angle.

The single-channel model for the scene image may be extended to encompass multichannel collections. For a repeatpass interferometric SAR image pair, the joint density function of a corresponding (registered) pixel pair $\underline{X}_{k}=\left[f_{k}, g_{k}\right]^{\mathrm{T}}$ may be described by a zero-mean complex circular Gaussian random vector with probability density function (pdf)

$$
p\left(\underline{X}_{k}\right)=\frac{1}{\pi^{2}|Q|^{2}} \exp \left(-\underline{X}_{k}^{\mathrm{H}} Q^{-1} \underline{X}_{k}\right)
$$

where $\underline{X}_{k}^{\mathrm{H}}$ is the complex conjugate transpose of $\underline{X}_{k}$ and $Q$ is the covariance matrix given by

$$
Q=E\left\{\underline{X}_{k} \underline{X}_{k}^{\mathrm{H}}\right\}=\left[\begin{array}{cc}
\sigma_{f}^{2} & \gamma \sigma_{f} \sigma_{g} \exp (j \Phi) \\
\gamma \sigma_{f} \sigma_{g} \exp (-j \Phi) & \sigma_{g}^{2}
\end{array}\right] .
$$

The terms $\sigma_{f}^{2}$ and $\sigma_{g}^{2}$ on the diagonal represent the mean backscatter power of the scene in the primary and repeat-pass collections, respectively. In general, $\sigma_{f}^{2} \neq \sigma_{g}^{2}$, due to the presence of scene disturbances that occur in the interval between collections as well as antenna-pointing errors arising in the data acquisition and radiometric miscalibration in the image formation. The term $\gamma \exp (j \Phi)$ is the complex cross channel correlation coefficient and encapsulates the additional information made available by the joint interferometric processing of the image pair. The interferometric phase $\Phi$ is related to the displacement between the primary and repeat-pass imaging flight tracks and also the terrain topography [15], [16]. The magnitude of the complex correlation coefficient $\gamma$, commonly referred to as the scene coherence, is a value between zero and one and is sensitive to changes in the observed amplitude and phase of the image resolution cells.

The coherence may be expressed as the product of a number of dominant contributions [3], [17]

$$
\gamma=\gamma_{\mathrm{SNR}} \gamma_{\text {base }} \gamma_{\mathrm{scene}} \gamma_{\mathrm{vol}} \gamma_{\text {proc }}
$$

The component $\gamma_{\mathrm{SNR}}$ is determined by the relative backscatter signal to radar receiver noise ratio in the interferometric image pair [3]. The baseline decorrelation $\gamma_{\text {base }}$ quantifies the decorrelation that arises due to mismatch in the acquisition geometries in the primary and repeat-pass collections [18]. The volume decorrelation $\gamma_{\mathrm{vol}}$ quantifies the decorrelation that arises when the images are acquired with a nonzero baseline and the scattering occurs from a volume such as a vegetated area [19]. The decorrelation depends the properties of the scattering volume such as the extinction coefficient, as well as the interferometric baseline and the polarization of the incident radiation [20]. The component $\gamma_{\text {proc }}$ quantifies the decorrelation arising from mismatch between the coherent acquisition apertures and image-formation processing stages used to produce the primary and repeat-pass imagery. The mismatch between the acquisition apertures and processing stages manifests as image domain misregistration as well as image defocusing [1]. The coherence term $\gamma_{\text {scene }}$ quantifies the decorrelation in the scene over the repeat-pass time interval, and is determined by the nature and extent of the various sources of scene change such as environmental effects, e.g., wind and rain as well as man-made scene disturbances. Through the careful design of the repeat-pass imaging geometry and appropriate interferometric processing steps including compensation for aperture and processor mismatch and image registration, it is possible to achieve $\gamma_{\mathrm{SNR}} \gamma_{\text {base }} \gamma_{\text {proc }} \gamma_{\mathrm{vol}} \approx 1$. In this case, the coherence $\gamma$ of the scene image will reflect the underlying true scene coherence $\gamma_{\text {scene }}$.

\section{SCEne-CHANGE MOdel}

Discriminating between those regions affected by the scene changes of interest (e.g., man-made changes) and those that are not can be achieved by formulating the detection problem in a hypothesis-testing framework. In this approach, the change-detection problem is to determine whether a pixel pair $\underline{X}_{k}=\left[f_{k}, g_{k}\right]^{\mathrm{T}}$ is a realization of a null hypothesis $H_{0}$ (scene changes of interest absent) or an alternative hypothesis $H_{1}$ (scene changes of interest present). Assuming that under $H_{0}$ and $H_{1}$ the jointly Gaussian model (1) is valid, then, in the most 
general case, the two hypotheses may be completely described by the covariance matrices

$$
Q_{0}=\left[\begin{array}{cc}
\sigma_{f}^{2} & \sigma_{f} \sigma_{g_{0}} \gamma_{0} \exp \left(j \Phi_{0}\right) \\
\sigma_{f} \sigma_{g_{0}} \gamma_{0} \exp \left(-j \Phi_{0}\right) & \sigma_{g_{0}}^{2}
\end{array}\right]
$$

under the unchanged-scene hypothesis $H_{0}$ and

$$
Q_{1}=\left[\begin{array}{cc}
\sigma_{f}^{2} & \sigma_{f} \sigma_{g_{1}} \gamma_{1} \exp \left(j \Phi_{1}\right) \\
\sigma_{f} \sigma_{g_{1}} \gamma_{1} \exp \left(j \Phi_{1}\right) & \sigma_{g_{1}}^{2}
\end{array}\right]
$$

under the changed-scene hypothesis $H_{1}$. The mean backscatter terms $\sigma_{g_{0}}^{2}$ and $\sigma_{g_{1}}^{2}$ describe the mean backscatter power in the repeat-pass image under the unchanged- and changedscene hypotheses, respectively. The scene coherence under the unchanged and changed hypotheses are given by $\gamma_{0}$ and $\gamma_{1}$, respectively, and, in general, the coherence values may assume any value between zero and one. Note that a different interferometric phase $\Phi_{1}$ has been used to characterize the changed scene compared to the unchanged-scene interferometric phase $\Phi_{0}$. This allows for the possibility of a common translational shift in the scene wherein all scatterers in a region are subject to a common height change (the measurement and characterization of such scene changes is commonly referred to in the literature as differential interferometric SAR processing [21] and has been used to monitor glacial movement and land subsidence). While the value of $\gamma_{0}$ may be derived from the observed imagery, the value for $\gamma_{1}$ depends on the source of the scene changes, the nature of the scene scattering, and the resolution of the imagery. In [3], a probabilistic model for the scatterer displacement between imaging observations was postulated and an rms displacement of approximately $20 \%$ of the radar wavelength was found to cause complete scene decorrelation. For X-band radars, man-made disturbances such as vehicle tracks across a field can potentially cause significant scatterer displacement, and hence a complete loss in coherence. However, for coarser resolution systems where the both changed and unchanged scattering is present in a resolution cell, a nonzero value of $\gamma_{1}$ would be more appropriate. In the subsequent analysis, an imaging system with fine resolution commensurate with the scene changes to be detected is considered, for which $\gamma_{1}=0$ will be assumed.

\section{Mean Backscatter-Power Change Statistic}

From $Q_{0}$ and $Q_{1}$, it is evident that one approach to discriminating between the changed- and unchanged-scene hypotheses is to estimate and compare the mean backscatter powers of the scene in the primary and repeat-pass imagery. Given a homogeneous $N$-pixel-pair local neighborhood under test, $\underline{X}_{k}=\left[f_{k}, g_{k}\right]^{\mathrm{T}}, k=1, \ldots, N$, the MLE of the mean backscatter power of the scene in the primary and repeat acquisitions is given by the sample average pixel intensities

$$
I_{f}=\frac{1}{N} \sum_{k=1}^{N}\left|f_{k}\right|^{2} \quad I_{g}=\frac{1}{N} \sum_{k=1}^{N}\left|g_{k}\right|^{2} .
$$

Provided the $N$-pixel realizations are independent, then $I_{f}$ and $I_{g}$ each have a Gamma distribution with order parameter $N$ of the form

$$
p\left(I \mid \sigma^{2}\right)=\frac{1}{\Gamma(N)}\left(\frac{N}{\sigma^{2}}\right)^{N} I^{N-1} \exp \left(-\frac{N I}{\sigma^{2}}\right)
$$

where $I$ and $\sigma^{2}$ are replaced by $I_{f}$ and $\sigma_{f}^{2}$ for the primary image and $I_{g}$ and $\sigma_{g}^{2}$ for the repeat-pass image. Note that, in practice, neighboring pixels are correlated due to the point spread function of the SAR processor. As a consequence, in an $N$-pixel sliding window, the effective number of independent pixels, commonly referred to as the effective number of looks (ENL), is less than $N$.

The sample estimates of the mean backscatter power may be compared by forming the following mean backscatter-power ratio proposed by Touzi et al. [22]:

$$
\hat{r}= \begin{cases}\hat{R}, & \text { if } \hat{R} \leq 1 \\ \hat{R}^{-1}, & \text { if } \hat{R}>1\end{cases}
$$

where $\hat{R}=I_{f} / I_{g}$. This statistic takes values between zero and one and its pdf has been derived in [22]

$p(\hat{r} \mid R)=\frac{\Gamma(2 N)}{\Gamma(N)^{2}}\left(\frac{R^{N}}{(\hat{r}+R)^{2 N}}+\frac{R^{-N}}{\left(\hat{r}+R^{-1}\right)^{2 N}}\right) \hat{r}^{N-1}$.

From the pdf of $\hat{r}$, analytical expressions for the probability of detection $P_{\mathrm{d}}$ and the probability of false alarm $P_{\mathrm{fa}}$ as a function of the detection threshold $T$ may be readily derived. The $P_{\mathrm{fa}}$ is given by

$$
\begin{aligned}
P_{\mathrm{fa}}= & \int_{0}^{\mathrm{T}} p\left(\hat{r} \mid R=R_{0}\right) d \hat{r} \\
= & \frac{\Gamma(2 N)}{\Gamma^{2}(N)} \int_{0}^{\mathrm{T}}\left(\frac{R_{0}^{N}}{\left(\hat{r}+R_{0}\right)^{2 N}}+\frac{R_{0}^{-N}}{\left(\hat{r}+R_{0}^{-1}\right)^{2 N}}\right) \hat{r}^{N-1} d \hat{r} \\
= & \frac{\Gamma(2 N) T^{N}}{\Gamma(N-1)}\left(\frac{1}{R_{0}^{N}}{ }_{1} F_{2}\left(2 N, N, N-1, \frac{T}{R_{0}}\right)\right. \\
& \left.\quad+R_{0}^{N}{ }_{1} F_{2}\left(2 N, N, N-1, T R_{0}\right)\right)
\end{aligned}
$$

where $R_{0}=\sigma_{f}^{2} / \sigma_{g 0}^{2}$ is the ratio of the mean backscattered power in the unchanged regions of the scene and ${ }_{1} F_{2}$ is the hypergeometric function [23]. The $P_{\mathrm{d}}$ has the same form as the $P_{\mathrm{fa}}$ in (10) but with $R_{0}$ replaced with $R_{1}=\sigma_{f}^{2} / \sigma_{g 1}^{2}$, which is the mean backscattered power ratio in the changed regions of the scene.

Fig. 1 shows simulated and theoretical receiver operating characteristic (ROC) curves (plots of the $P_{\mathrm{d}}$ versus $P_{\mathrm{fa}}$ ) for the ratio detector $\hat{r}$ obtained with $R_{0}=0 \mathrm{~dB}$ and $R_{1}=$ $1,3,5$, and $10 \mathrm{~dB}$, and $N=7$. It is clear that for small changes in the mean backscatter power, the detector suffers from a significant false-alarm rate. To achieve a probability of detection of 0.7 for a mean backscatter-power change of $3 \mathrm{~dB}$, the associated probability of false alarm is unacceptably high at 0.4 . The false-alarm rate may be reduced by increasing the window size $N$ used in computing $I_{f}$ and $I_{g}$. However, this improvement in detection performance is only realized if the scene's mean backscatter power over the estimation window 


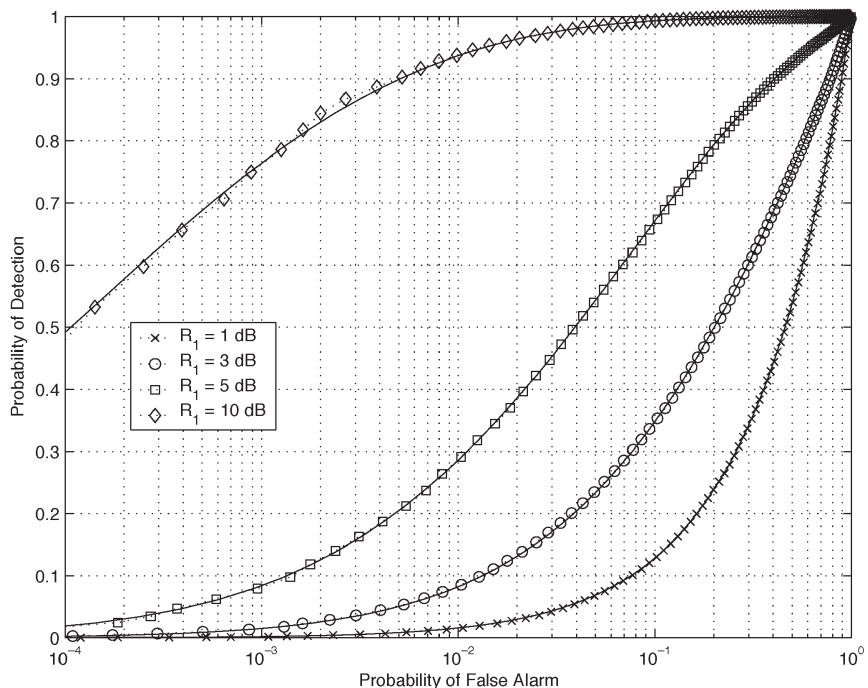

Fig. 1. Simulated (indicated by the symbols) and theoretical ROC curves for the intensity-ratio change statistic obtained with $N=7$ and mean backscatterpower changes of $1,3,5$, and $10 \mathrm{~dB}$.

is locally stationary. When applying the ratio detector, the estimation window must be commensurate with the size of the scene disturbances to be detected otherwise the changestatistic local estimate contains contributions from changed and unchanged image pixels.

\section{Sample Coherence Change Statistic}

The scene-change models specified by (4) and (5) indicate that regions of man-made scene disturbance may be detected as areas of zero coherence against undisturbed areas that are characterized by some degree of partial coherence. A simple threshold detector based on the sample coherence evaluated over a local neighborhood of $N$ pixels $\underline{X}_{k}=\left[f_{k}, g_{k}\right]^{\mathrm{T}}, k=$ $1, \ldots, N$

$$
\hat{\gamma}=\frac{\left|\sum_{k=1}^{N} f_{k} g_{k}^{*}\right|}{\sqrt{\sum_{k=1}^{N}\left|f_{k}\right|^{2} \sum_{k=1}^{N}\left|g_{k}\right|^{2}}}
$$

may be used to detect the changed regions of the scene.

The statistics of the sample coherence have been extensively studied in the literature [24], [25]. The density function of the sample coherence for a general repeat-pass image pair with true underlying coherence $\gamma$ is given by

$$
\begin{aligned}
p(\hat{\gamma} \mid \gamma, N)=2(N-1)\left(1-\gamma^{2}\right)^{N} & \hat{\gamma}\left(1-\hat{\gamma}^{2}\right)^{(N-2)} \\
& \times{ }_{2} F_{1}\left(N, N ; 1 ; \gamma^{2} \hat{\gamma}^{2}\right)
\end{aligned}
$$

where ${ }_{2} F_{1}$ is the Gauss hypergeometric function. The density function is dependent on the underlying scene coherence $\gamma$ and the number of independent pixels $N$ used in the estimation window. Note that, like the sample mean backscatter-power estimate discussed in the previous section, in practice, $N$ in (12) refers to the ENL, i.e., the effective number of independent realizations of the random process $\underline{X}$ in the sliding estimation window.

The performance of the sample coherence detector (11) may be evaluated by computing the associated ROC curve, which

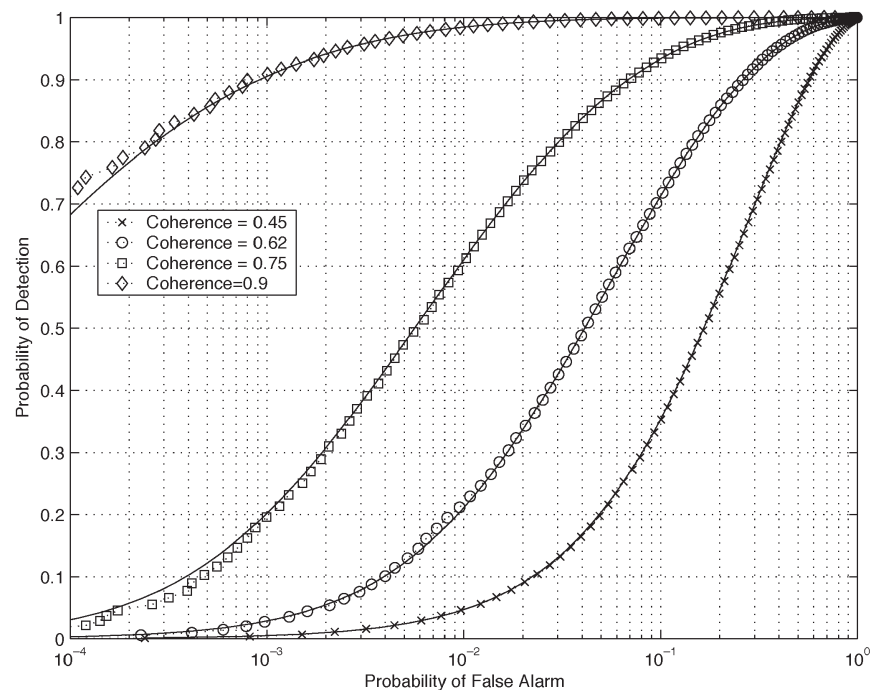

Fig. 2. Simulated (indicated by the symbols) and theoretical ROC curves for sample coherence change statistic obtained with $\gamma_{0}=0.45,0.62,0.75$, and $0.9, \gamma_{1}=0$, and an estimation-window size of $N=7$ independent pixels.

indicates, for a given detection threshold $T$, the probability of detecting a changed pixel $P_{\mathrm{d}}$ and the corresponding probability of a false alarm $P_{\text {fa }}$, i.e.,

$$
\begin{aligned}
P_{\mathrm{d}} & =\int_{0}^{\mathrm{T}} p(\hat{\gamma} \mid \gamma=0) d \hat{\gamma} \\
P_{\mathrm{fa}} & =\int_{0}^{\mathrm{T}} p\left(\hat{\gamma} \mid \gamma=\gamma_{0}\right) d \hat{\gamma} .
\end{aligned}
$$

Substituting the density function given in (12) into (13) and (14) gives the following:

$$
\begin{aligned}
P_{\mathrm{fa}}= & \frac{2(N-1)\left(1-\gamma_{0}^{2}\right)^{N}}{\Gamma(N) \Gamma(N-1)} \sum_{k=0}^{N-2}\left(\begin{array}{c}
N-2 \\
k
\end{array}\right)(-1)^{N-2-k} \\
& \times \sum_{l=0}^{\infty}\left[\frac{\Gamma(N+l)}{\Gamma(l+1)}\right]^{2} \gamma_{0}^{2 l} \frac{T^{2 N+2 l-2-2 k}}{2 N+2 l-2-2 k} \\
P_{\mathrm{d}}= & 2(N-1)^{2} \sum_{k=0}^{N-2}\left(\begin{array}{c}
N-2 \\
k
\end{array}\right)(-1)^{N-2-k} \frac{T^{2 N-2-2 k}}{2 N-2-2 k} .
\end{aligned}
$$

Fig. 2 shows the ROC curves for $N=7, \gamma_{0}=0.45$, $0.62,0.75$, and 0.9. A comparison of Fig. 2 with the ROC curve for the backscatter-power ratio change statistic in Fig. 1 shows that for an estimation-window size of $N=7$ pixels, an equivalent ROC performance of $P_{\mathrm{d}}=0.7$ at $P_{\mathrm{fa}}=0.1$ occurs for a magnitude change of $5 \mathrm{~dB}$ (Fig. 1) or an unchanged sample coherence of $\gamma_{0}=0.62$ (Fig. 2).

The detection performance of the sample coherence change statistic may be improved by increasing the estimation-window size. The window size, however, must be commensurate with the size of the scene disturbances to be detected; otherwise, the sample coherence contains contributions from both changed and unchanged pixels. 


\section{New Log-Likelihood Change Statistic}

The sample coherence and mean backscatter ratio discussed in the previous sections have been used extensively in the literature as both change statistics and also in scene classification problems. In [2], the sample coherence and mean backscatter ratio are used to detect changes in repeat-pass European Remote Sensing 1 SAR imagery. It was found that the areas of disturbance identified by each method did not necessarily agree and each method gives complementary characterizations of scene changes. In the context of change detection, this presents problems in fusing the detections from the two change statistics to achieve a single combined detection list. A further point to note from the analysis presented in the previous sections is that the sample coherence and mean backscatter ratio are susceptible to high false-alarm rates unless there is a significant change in the mean backscatter power or the $H_{0}$ scene coherence $\gamma_{0}$ is high. The high false-alarm rate may be mitigated if extensive averaging is carried out. Increased averaging, however, degrades the resolution of the change statistic. Furthermore, the estimation window must be commensurate with the size of the scene changes; otherwise, the anticipated performance improvement is not realized.

An alternative approach to discriminating between the $H_{0}$ and $H_{1}$ hypotheses given a local neighborhood of $N$ independent pixels $\underline{X}_{k}=\left[f_{k}, g_{k}\right]^{\mathrm{T}}, k=1, \ldots, N$, is to form the likelihood ratio given by

$$
\begin{aligned}
L & =\frac{p\left(\underline{X}_{1}, \underline{X}_{2}, \ldots, \underline{X}_{N} ; H_{0}\right)}{p\left(\underline{X}_{1}, \underline{X}_{2}, \ldots, \underline{X}_{N} ; H_{1}\right)} \\
& =\prod_{k=1}^{N} \frac{p\left(\underline{X}_{k} ; H_{0}\right)}{p\left(\underline{X}_{k} ; H_{1}\right)} .
\end{aligned}
$$

Substituting $Q=Q_{0}$ in (1) gives the density function $p\left(\underline{X}_{k} ; H_{0}\right)$, and $Q=Q_{1}$ gives $p\left(\underline{X}_{k} ; H_{1}\right)$. Thus, (17) becomes

$$
L=\left(\frac{\left|Q_{1}\right|}{\left|Q_{0}\right|}\right)^{N} \exp \left(-\operatorname{Tr}\left\{\left(Q_{0}^{-1}-Q_{1}^{-1}\right) \sum_{k=1}^{N} \underline{X}_{k} \underline{X}_{k}^{\mathrm{H}}\right\}\right)
$$

where $\operatorname{Tr}\{A\}$ denotes the trace of matrix $A$. Taking the log of (18) and ignoring the constant term yields the following decision statistic for discriminating between the two hypotheses:

$$
\begin{aligned}
z & =\operatorname{Tr}\left\{\left(Q_{0}^{-1}-Q_{1}^{-1}\right) \sum_{k=1}^{N} \underline{X}_{k} \underline{X}_{k}^{\mathrm{H}}\right\} \\
& =\operatorname{Tr}\left\{Q_{d} G\right\}
\end{aligned}
$$

where $G=\sum_{k=1}^{N} \underline{X}_{k} \underline{X}_{k}^{\mathrm{H}}$ and $Q_{d}=Q_{0}^{-1}-Q_{1}^{-1}$. Note that $G$ is the sample covariance formed from the $N$-pixel area under test, and under the jointly Gaussian scattering assumption, $G$ has a complex Wishart distribution [26]. Note also that the matrices $Q_{0}$ and $Q_{1}$ describing the $H_{0}$ and $H_{1}$ hypotheses consist of a number of unknown parameters, thus the hypotheses are composite, and $z$ in (19) is referred to as a clairvoyant detector [11]. A clairvoyant detector yields the optimal detection performance achievable given perfect knowledge of the unknown parameters. In practice, suboptimal tests are implemented in which the unknown parameters are specified by their MLEs to form a generalized likelihood-ratio test (GLRT) [11].

\section{PDF OF LOG-Likelihood Change STATISTIC}

Assuming perfect knowledge of the unknown parameters in $H_{0}$ and $H_{1}$, the pdf of the clairvoyant detector $z$ in (19) may be derived by applying a linear transform $P$ to the image pixelpair vector $\underline{X}_{k}=\left[f_{k}, g_{k}\right]^{\mathrm{T}}$. The matrix $P$ is chosen such that it diagonalizes the rank-two matrix $Q_{d}$

$$
P^{\mathrm{H}} Q_{d} P=\left[\begin{array}{cc}
\lambda_{1} & 0 \\
0 & \lambda_{2}
\end{array}\right]=\operatorname{diag}\left(\lambda_{1}, \lambda_{2}\right)
$$

where $\lambda_{1}$ and $\lambda_{2}$ are the eigenvalues of $Q_{d}$ and the columns of $P$ are the corresponding eigenvectors [27]. Furthermore, it may be shown that, given the forms for $Q_{0}$ in (4) and $Q_{1}$ in (5), one of the eigenvectors is negative while the other is positive. In the following analysis, it will be assumed that the first eigenvalue is negative and $\lambda_{1}$ will be taken to mean the absolute value of the first eigenvalue (similar forms for the density functions as those derived in the following analysis may be obtained for the case when the second eigenvalue is negative). Defining the new transform variables $u$ and $v$ such that

$$
\underline{X}=\left[\begin{array}{l}
f \\
g
\end{array}\right]=P\left[\begin{array}{l}
u \\
v
\end{array}\right]
$$

the decision statistic may subsequently be written as

$$
\begin{aligned}
z & =\operatorname{Tr}\left\{Q_{d} \sum_{k=1}^{N}\left(\left[\begin{array}{l}
f_{k} \\
g_{k}
\end{array}\right]\left[\begin{array}{ll}
f_{k}^{*} & g_{k}^{*}
\end{array}\right]\right)\right\} \\
& =\operatorname{Tr}\left\{P^{\mathrm{H}} Q_{d} P \sum_{k=1}^{N}\left(\left[\begin{array}{l}
u_{k} \\
v_{k}
\end{array}\right]\left[\begin{array}{ll}
u_{k}^{*} & v_{k}^{*}
\end{array}\right]\right)\right\} \\
& =-\lambda_{1} \sum_{k=1}^{N}\left|u_{k}\right|^{2}+\lambda_{2} \sum_{k=1}^{N}\left|v_{k}\right|^{2} .
\end{aligned}
$$

In general, under the transform $P$, the new transform variables $u$ and $v$ are dependent zero-mean circular complex Gaussian random variables with a covariance matrix of the form

$$
C=\left[\begin{array}{cc}
C_{11} & \sqrt{C_{11} C_{22}} \rho e^{j \theta} \\
\sqrt{C_{11} C_{22}} \rho e^{-j \theta} & C_{22}
\end{array}\right]
$$

where

$$
C= \begin{cases}P^{\mathrm{H}} Q_{0} P, & \text { for } u \text { and } v \text { realizations of } H_{0} \\ P^{\mathrm{H}} Q_{1} P, & \text { for } u \text { and } v \text { realizations of } H_{1}\end{cases}
$$

Therefore, the variables $a=\lambda_{1} \sum_{k=1}^{N}\left|u_{k}\right|^{2}$ and $b=$ $\lambda_{2} \sum_{k=1}^{N}\left|v_{k}\right|^{2}$ that form the decision statistic (22) are mutually dependent chi-square random variables with $2 N$ degrees of freedom [11]. The joint density function of the two dependent chi-square random variables has been derived by Lee et al. [28]. Applying the result of Lee et al., the joint density function of $a$ and $b$ may be written as

$$
\begin{aligned}
p(a, b)=\frac{\left(1-\rho^{2}\right)^{N}(a b)^{N-1} \exp \left\{-\left(\frac{a}{\alpha}+\frac{b}{\beta}\right)\right\}}{(\alpha \beta)^{N} \Gamma^{2}(N)} \\
\times{ }_{1} F_{2}\left(1 ; 1, N ; \frac{a b \rho^{2}}{\alpha \beta}\right)
\end{aligned}
$$


where ${ }_{1} F_{2}$ is a hypergeometric function and

$$
\begin{aligned}
& \alpha=\lambda_{1} C_{11}\left(1-\rho^{2}\right) \\
& \beta=\lambda_{2} C_{22}\left(1-\rho^{2}\right) .
\end{aligned}
$$

The density function of the decision statistic $z=-a+b$ may subsequently be found from (25) by a direct evaluation of the following integrals:

$$
p(z)= \begin{cases}\int_{0}^{\infty} p(b-z, b) d b, & \text { for } z \leq 0 \\ \int_{0}^{\infty} p(a, a+z) d a, & \text { for } z>0\end{cases}
$$

where the lower limit in each integral arises because $\lambda_{1} \geq$ 0 and $\lambda_{2} \geq 0$, and so $a \geq 0$ and $b \geq 0$. Evaluating (28) for $z \leq 0$ gives

$$
\begin{array}{r}
p(z)=\frac{\left(1-\rho^{2}\right)^{N} e^{\left(\frac{z}{\alpha}\right)}}{\Gamma(N)(\alpha \beta)^{N}} \sum_{k=0}^{\infty}\left[\mu _ { k } \sum _ { p = 0 } ^ { N + k - 1 } \left[\left(\begin{array}{c}
N+k-1 \\
p
\end{array}\right)\right.\right. \\
\left.\left.\times \frac{\Gamma(2 k+2 N-p-1)}{\nu^{2 k+2 N-p-1}}(-z)^{p}\right]\right]
\end{array}
$$

and, for $z>0$

$$
\begin{array}{r}
p(z)=\frac{\left(1-\rho^{2}\right)^{N} e^{\left(\frac{-z}{\beta}\right)}}{\Gamma(N)(\alpha \beta)^{N}} \sum_{k=0}^{\infty}\left[\mu _ { k } \sum _ { p = 0 } ^ { N + k - 1 } \left[\left(\begin{array}{c}
N+k-1 \\
p
\end{array}\right)\right.\right. \\
\left.\left.\times \frac{\Gamma(2 k+2 N-p-1)}{\nu^{2 k+2 N-p-1}}(-z)^{p}\right]\right] .
\end{array}
$$

The derivation of these results is given in Appendix I. Note that (29) and (30) are general expressions for the density function of $z$. To obtain $p\left(z ; H_{0}\right)$ and $p\left(z ; H_{1}\right)$, the appropriate values for $C_{11}, C_{22}$, and $\rho$, defined in (23) and (24), are used.

\section{A. Special Case of Equal Mean Backscatter Power}

Scene disturbances arising from subtle man-made changes, e.g., disturbances due to the passage of vehicles through a scene, are commonly characterized by an absence of any measurable change in the mean backscatter power coupled with a complete loss in scene coherence. In such cases, for calibrated image pairs, a simpler closed-form solution for the density function of the clairvoyant decision statistic may be derived. Substituting $\sigma_{f}^{2}=\sigma_{g 0}^{2}=\sigma_{g 1}^{2}$ into (4) and (5), the $Q_{d}$ matrix may be written as

$$
\begin{aligned}
Q_{d} & =Q_{0}^{-1}-Q_{1}^{-1} \\
& =\frac{\gamma_{0}}{\sigma_{f}^{2}\left(1-\gamma_{0}^{2}\right)}\left[\begin{array}{cc}
\gamma_{0} & -\exp \left(j \Phi_{0}\right) \\
-\exp \left(-j \Phi_{0}\right) & \gamma_{0}
\end{array}\right] .
\end{aligned}
$$

The eigenvalues of $Q_{d}$ are given by

$$
\lambda_{1}=-\frac{\gamma_{0}}{\sigma_{f}^{2}\left(1+\gamma_{0}\right)} \quad \lambda_{2}=\frac{\gamma_{0}}{\sigma_{f}^{2}\left(1-\gamma_{0}\right)}
$$

and the corresponding eigenvectors form the columns of the transform matrix $P$ that diagonalizes $Q_{d}$

$$
P=\frac{1}{\sqrt{2}}\left[\begin{array}{cc}
\exp \left(j \Phi_{0}\right) & -\exp \left(j \Phi_{0}\right) \\
1 & 1
\end{array}\right] .
$$

The transform variables $u$ and $v$, obtained by applying $P$ to the observations $f$ and $g$, are now independent zero-mean complex Gaussian random variables with a covariance matrix given by

$$
\begin{aligned}
C & =\left[\begin{array}{cc}
C_{11} & 0 \\
0 & C_{22}
\end{array}\right] \\
& = \begin{cases}P^{\mathrm{H}} Q_{0} P=\sigma_{f}^{2}\left[\begin{array}{cc}
1+\gamma_{0} & 0 \\
0 & 1-\gamma_{0}
\end{array}\right] & H_{0} \\
r P^{\mathrm{H}} Q_{1} P=\sigma_{f}^{2}\left[\begin{array}{ll}
1 & 0 \\
0 & 1
\end{array}\right] & H_{1} .\end{cases}
\end{aligned}
$$

The decision statistic, thus, takes the form

$$
z=-\lambda_{1} \sum_{k=1}^{N}\left|u_{k}\right|^{2}+\lambda_{2} \sum_{k=1}^{N}\left|v_{k}\right|^{2}
$$

where $\lambda_{1}$ is taken to mean the absolute value of the first eigenvalue of $Q_{d}$. The variables $a=\lambda_{1} \sum\left|u_{k}\right|^{2}$ and $b=\lambda_{1} \sum\left|v_{k}\right|^{2}$ in this case are mutually independent chi-square random variables with density functions

$$
\begin{aligned}
& p(a)=\frac{1}{\Gamma(N) \lambda_{1} C_{11}}\left(\frac{a}{\lambda_{1} C_{11}}\right)^{N-1} \exp \left(-\frac{a}{\lambda_{1} C_{11}}\right) \\
& p(b)=\frac{1}{\Gamma(N) \lambda_{2} C_{22}}\left(\frac{b}{\lambda_{2} C_{22}}\right)^{N-1} \exp \left(-\frac{b}{\lambda_{2} C_{22}}\right) .
\end{aligned}
$$

The density function of the log-likelihood decision statistic $z=-a+b$ may subsequently be found by a direct evaluation of the integrals

$$
p(z)= \begin{cases}\int_{0}^{\infty} p(a) p(a+z) d a, & z>0 \\ \int_{0}^{\infty} p(b-z) p(b) d b, & z \leq 0 .\end{cases}
$$

Evaluating (38), for $z \leq 0$, gives

$$
\begin{aligned}
p(z)=\frac{\exp \left(\frac{z}{\lambda_{1} C_{11}}\right)}{\Gamma^{2}(N)\left(\lambda_{1} C_{11} \lambda_{2} C_{22}\right)^{N}} \\
\quad \times \sum_{p=0}^{N-1}\left[\left(\begin{array}{c}
N-1 \\
p
\end{array}\right) \frac{\Gamma(2 N-p-1)}{\nu^{2 N-p-1}}(-z)^{p}\right]
\end{aligned}
$$

and similarly, for $z>0$

$$
\begin{aligned}
p(z)=\frac{\exp \left(\frac{-z}{\lambda_{2} C_{22}}\right)}{\Gamma^{2}(N)\left(\lambda_{1} C_{11} \lambda_{2} C_{22}\right)^{N}} \\
\quad \times \sum_{p=0}^{N-1}\left[\left(\begin{array}{c}
N-1 \\
p
\end{array}\right) z^{p} \frac{\Gamma(2 N-p-1)}{\nu^{2 N-p-1}}\right] .
\end{aligned}
$$

These pdfs are derived in Appendix II. Equations (39) and (40) are simple closed-form polynominal solutions for the pdfs of the decision statistic. Note that these equations are general expressions of the density function of $z$. To obtain $p\left(z ; H_{0}\right)$ and $p\left(z ; H_{1}\right)$, the appropriate values for $C_{11}$ and $C_{22}$, defined in (34), are used. Fig. 3 shows the density function of the decision statistic $z$ for both $H_{0}$ and $H_{1}$ obtained by direct computation of (39) and (40) and also via a simulation with $\gamma_{0}=0.62$ and $N=7$. 


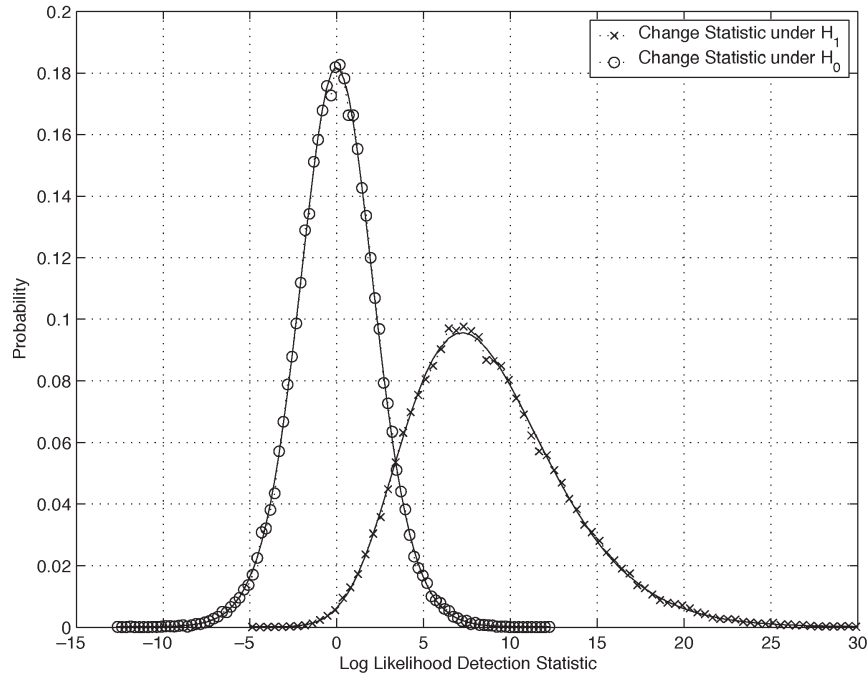

Fig. 3. Simulated and theoretical PDF curves for the log-likelihood change statistic obtained with $\gamma_{0}=0.62, \sigma_{f}^{2}=\sigma_{g 0}^{2}=\sigma_{g 1}^{2}$, and an estimationwindow size of $N=7$ independent pixels.

\section{Detection Performance of the Clairvoyant Detector}

Expressions for the probability of detection and false alarm for the clairvoyant log-likelihood detector, as a function of the decision threshold $T$, may be derived using the pdfs $p\left(z ; H_{0}\right)$ and $p\left(z ; H_{1}\right)$ given in the previous section by evaluating the integrals

$$
\begin{aligned}
P_{\mathrm{fa}} & =\int_{T}^{\infty} p\left(z ; H_{0}\right) d z \\
P_{\mathrm{d}} & =\int_{T}^{\infty} p\left(z ; H_{1}\right) d z .
\end{aligned}
$$

Using (29) and (30), $P_{\mathrm{d}}$ and $P_{\mathrm{fa}}$ both take the following general form for $T>0$ :

$$
\begin{aligned}
& P_{\mathrm{d} \text { or fa }} \\
& \begin{array}{l}
=\frac{\left(1-\rho^{2}\right)^{N}}{\Gamma(N)(\alpha \beta)^{N}} \\
\quad \times \sum_{k=0}^{\infty}\left[\mu _ { k } \sum _ { p = 0 } ^ { N + k - 1 } \left[\left(\begin{array}{c}
N+k-1 \\
p
\end{array}\right) \frac{\Gamma(2 N+2 k-p-1)}{\nu^{2 k+2 N-p-1}}\right.\right. \\
\left.\left.\times \int_{T}^{\infty} e^{\left(-\frac{z}{\beta}\right)}(z)^{p} d z\right]\right] \\
=\frac{\left(1-\rho^{2}\right)^{N}}{\Gamma(N)(\alpha \beta)^{N}} \\
\quad \times \sum_{k=0}^{\infty}\left[\mu _ { k } \sum _ { p = 0 } ^ { N + k - 1 } \left[\left(\begin{array}{c}
N+k-1 \\
p
\end{array}\right) \beta^{p+1} \frac{\Gamma(2 N+2 k-p-1)}{\nu^{2 k+2 N-p-1}}\right.\right. \\
\left.\left.\times \Gamma_{\text {inc }}\left(p+1, \frac{T}{\beta}\right)\right]\right]
\end{array}
\end{aligned}
$$

where $\Gamma_{\text {inc }}$ is the complementary incomplete gamma function given by

$$
\Gamma_{\text {inc }}(a, t)=\int_{t}^{\infty} \exp (-x) x^{a-1} d x .
$$

For $T \leq 0$, the integrals giving the probability of false alarm (41) and probability of detection (42) must be partitioned into an integral from $T$ to 0 , where $p(z)$ is given by (29), plus an integral from 0 to $\infty$, where $p(z)$ is given by (30). The probability of detection and probability of false alarm, thus, both take the following form for $T<0$

$$
\begin{aligned}
& P_{\text {dorfa }} \\
& =\frac{\left(1-\rho^{2}\right)^{N}}{\Gamma(N)(\alpha \beta)^{N}} \\
& \times \sum_{k=0}^{\infty}\left[\mu _ { k } \sum _ { p = 0 } ^ { N + k - 1 } \left[\left(\begin{array}{c}
N+k-1 \\
p
\end{array}\right) \frac{\Gamma(2 N+2 k-p-1)}{\nu^{2 k+2 N-p-1}}\right.\right. \\
& \left.\left.\times\left[\int_{0}^{\infty} e^{\left(-\frac{z}{\beta}\right)}(z)^{p} d z+\int_{T}^{0} e^{\left(\frac{z}{\alpha}\right)}(-z)^{p} d z\right]\right]\right] \\
& =\frac{\left(1-\rho^{2}\right)}{\Gamma(N)(\alpha \beta)^{N}} \\
& \times \sum_{k=0}^{\infty}\left[\mu _ { k } \sum _ { p = 0 } ^ { N + k - 1 } \left[\left(\begin{array}{c}
N+k-1 \\
p
\end{array}\right) \frac{\Gamma(2 N+2 k-p-1)}{\nu^{2 k+2 N-p-1}}\right.\right. \\
& \times\left[\beta^{p+1} \Gamma(p+1)+\alpha^{p+1}\right. \\
& \left.\left.\left.\times\left(\Gamma(p+1)-\Gamma_{\text {inc }}\left(p+1, \frac{-T}{\alpha}\right)\right)\right]\right]\right]
\end{aligned}
$$

where the following relation has been used [23]:

$$
\int_{0}^{t} \exp (-x) x^{a-1} d x=\Gamma(a)-\Gamma_{\mathrm{inc}}(a, t) .
$$

The values for $C_{11}, C_{22}$, and $\rho$ used in the computation of $\mu_{k}$, $\alpha$, and $\beta$, respectively, in (43) and (45) are defined in (24) for each hypothesis.

Fig. 4 indicates the detection performance of the clairvoyant change statistic $z$ for a change scenario with $\gamma_{0}=0.62$ and an increasing mean backscatter-power ratio with $\sigma_{g 1}^{2} / \sigma_{g 0}^{2} \equiv$ $0,1,3,5$, and $10 \mathrm{~dB}$. Comparing the ROC curve in Fig. 4 associated with change scenario $\gamma_{0}=0.62$ and $\sigma_{g 1}^{2} / \sigma_{g 0}^{2} \equiv 0 \mathrm{~dB}$ and the corresponding ROC curve for the sample coherence in Fig. 2 shows that the log-likelihood change statistic yields significant improvements in detection performance. At a $P_{\mathrm{d}}$ of 0.7 , the $P_{\mathrm{fa}}$ for the sample coherence change statistic is 0.1 , while for the log-likelihood change statistic, the $P_{\mathrm{fa}}=$ 0.006 , which is a reduction in the false-alarm rate of over 


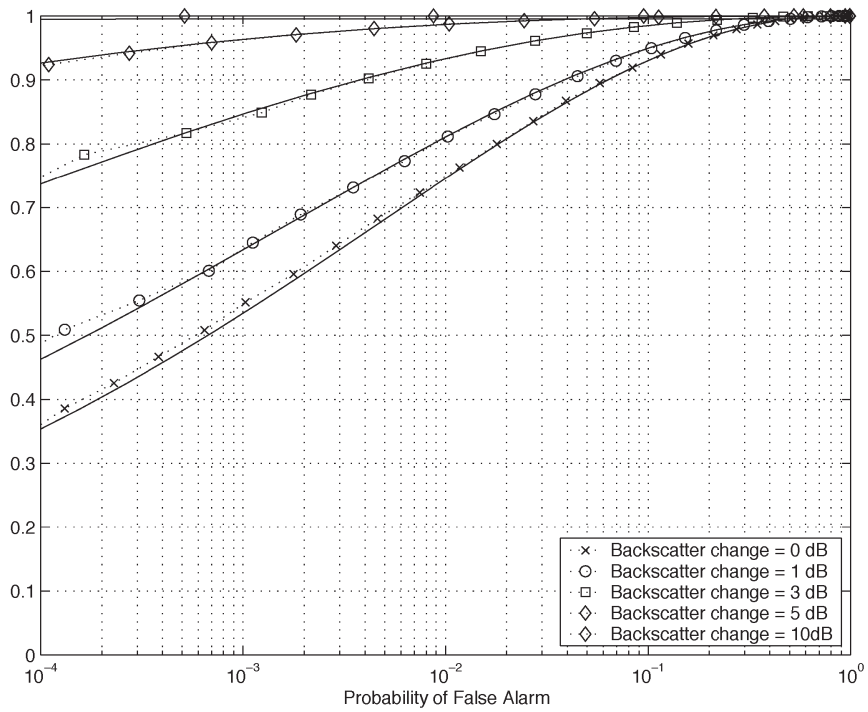

Fig. 4. Simulated and theoretical ROC curves for the log-likelihood statistic obtained with $N=7, \gamma_{0}=0.62$, and $\sigma_{g 1}^{2} / \sigma_{g 0}^{2}=0,1,3,5$, and $10 \mathrm{~dB}$.

an order of magnitude. As the mean backscatter-power ratio $\sigma_{g 1}^{2} / \sigma_{g 0}^{2}$ is increased, the detection performance of the loglikelihood change statistic improves, as illustrated in Fig. 4. For a mean backscatter-power ratio of $\sigma_{g 1}^{2} / \sigma_{g 0}^{2} \equiv 1 \mathrm{~dB}$ and $P_{\mathrm{d}}=0.7$, the log-likelihood change statistic $P_{\mathrm{fa}}=0.0025$, compared to $P_{\mathrm{fa}}=0.6$ for the mean backscatter-power ratio change statistic indicated in Fig. 1. Unlike the change statistics $\hat{r}$ and $\hat{\gamma}$, which are limited to detecting changes in only the mean backscatter power and the coherence, respectively, the loglikelihood change statistic uses both indicators of scene change to distinguish between the $H_{0}$ and $H_{1}$ hypotheses. It should be noted that the detection performance of the sample coherence clairvoyant change statistic $z$ and the mean backscatter power is being measured using the definitions of change and no change encapsulated in the $H_{1}$ and $H_{0}$ hypotheses.

\section{A. Detection Performance Under Equal Backscatter Power}

In the case where the primary and repeat-pass images have equal mean backscatter power under both $H_{0}$ and $H_{1}$, substituting (39) and (40) into (41) and (42) yields the following simpler general forms for the probability of detection and probability of false alarm. For $T>0$

$$
\begin{aligned}
P= & \frac{1}{\Gamma^{2}(N)\left(\lambda_{1} C_{11} \lambda_{2} C_{22}\right)^{N}} \\
& \times \sum_{p=0}^{N-1}\left[\left(\begin{array}{c}
N-1 \\
p
\end{array}\right) \frac{\Gamma(2 N-p-1)}{\nu^{2 N-p-1}} \int_{T}^{\infty} \exp \left(\frac{-z}{\lambda_{2} C_{22}}\right) z^{p} d z\right] \\
= & \frac{1}{\Gamma^{2}(N)\left(\lambda_{1} C_{11} \lambda_{2} C_{22}\right)^{N}} \\
& \times \sum_{p=0}^{N-1}\left[\left(\begin{array}{c}
N-1 \\
p
\end{array}\right)\left(\lambda_{2} C_{22}\right)^{p+1} \frac{\Gamma(2 N-p-1)}{\nu^{2 N-p-1}} \Gamma_{\mathrm{inc}}\right. \\
& \left.\quad \times\left(p+1, \frac{T}{\lambda_{2} C_{22}}\right)\right]
\end{aligned}
$$

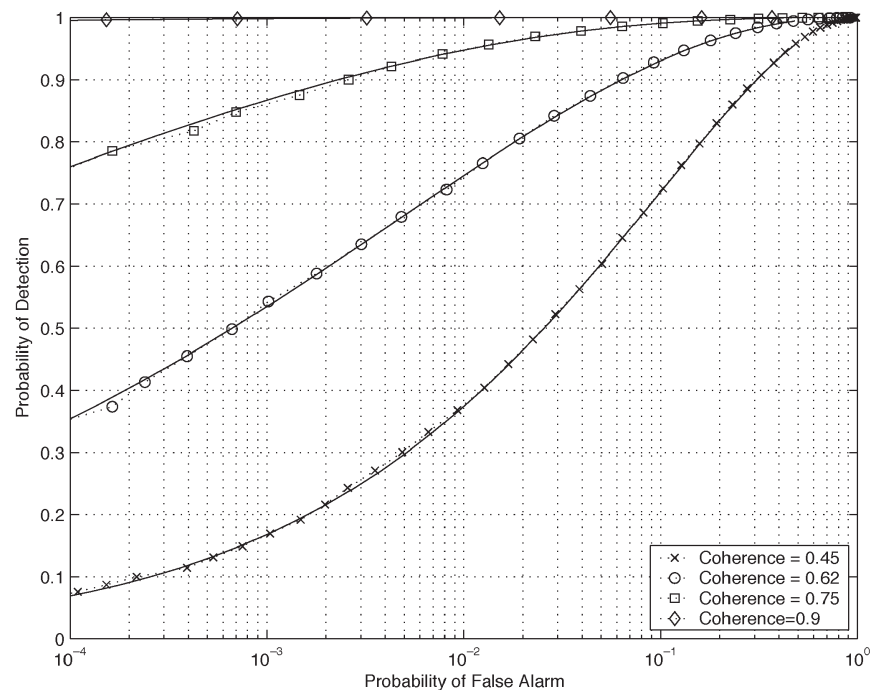

Fig. 5. Simulated and theoretical ROC curves for log-likelihood statistic obtained with $\gamma_{0}=0.45,0.62,0.75$, and $0.9, N=7$, and $\sigma_{f}^{2}=\sigma_{g 0}^{2}=\sigma_{g 1}^{2}$.

and for $T \leq 0$

$$
\begin{aligned}
& P= \frac{1}{\Gamma^{2}(N)\left(\lambda_{1} C_{11} \lambda_{2} C_{22}\right)^{N}} \\
& \times \sum_{p=0}^{N-1}\left[\left(\begin{array}{c}
N-1 \\
p
\end{array}\right) \frac{\Gamma(2 N-p-1)}{\nu^{2 N-p-1}}\right. \\
& \times\left[\int_{T}^{0} \exp \left(\frac{-z}{\lambda_{1} C_{11}}\right)(-z)^{p} d z\right. \\
&\left.\left.=\frac{+\int_{0}^{\infty} \exp \left(\frac{-z}{\lambda_{2} C_{22}}\right)(z)^{p} d z}{\Gamma^{2}(N)\left(\lambda_{1} C_{11} \lambda_{2} C_{22}\right)^{N}}\right]\right] \\
& \times \sum_{p=0}^{N-1}\left[\left(\begin{array}{c}
N-1 \\
p
\end{array}\right) \frac{\Gamma(2 N-p-1)}{\nu^{2 N-p-1}}\right. \\
& \times\left[\begin{array}{c}
\left(\lambda_{2} C_{22}\right)^{p+1} \Gamma(p+1)+\left(\lambda_{1} C_{11}\right)^{p+1} \\
\left.\left.\times \Gamma_{\mathrm{inc}}\left(p+1, \frac{-T}{\lambda_{1} C_{11}}\right)\right]\right] .
\end{array}\right.
\end{aligned}
$$

Fig. 5 indicates the detection performance of the loglikelihood change statistic under the equal mean backscatterpower scenario with values of $\gamma_{0}=0.45,0.62,0.75$, and 0.9 , and $N=7$. Comparing this with the detection performance of the sample coherence, as illustrated in Fig. 2, under the same change scenarios, it is shown that the log likelihood yields significant improvements in the detection performance, typically in excess of an order of magnitude.

\section{Generalized Log-Likelihood Change Statistic}

The ROC curves in Figs. 4 and 5 describe the performance of a clairvoyant detector in which perfect knowledge 


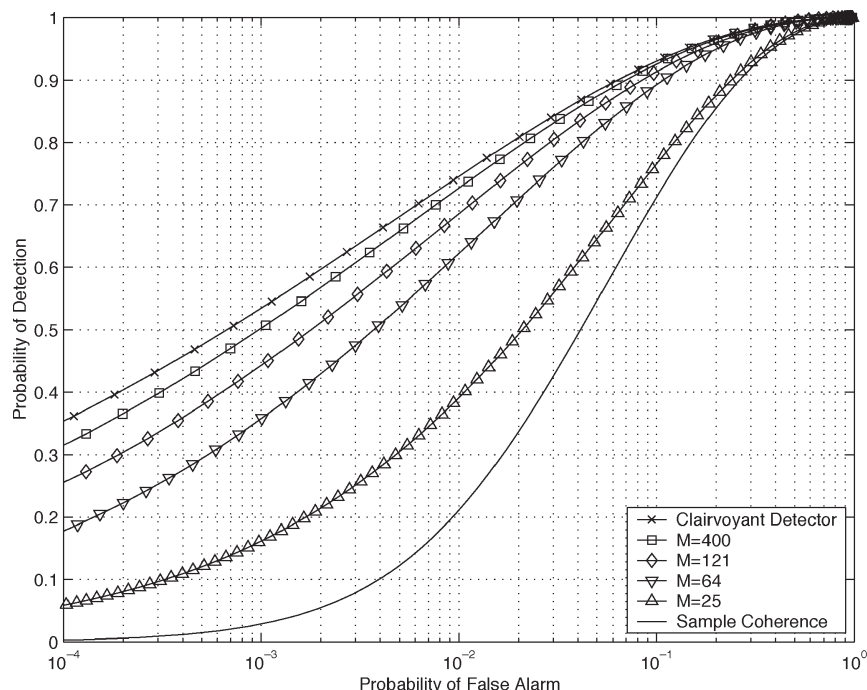

Fig. 6. Average ROC curves for the GLRT change statistic obtained using Monte Carlo simulation techniques with unchanged backscatter power $\sigma_{f}^{2}=\sigma_{g 0}^{2}=\sigma_{g 1}^{2}$, unchanged coherence of $\gamma_{0}=0.62$, and $N=7$.

of the unknown covariance matrices $Q_{0}$ and $Q_{1}$ has been assumed. While such a detector is unrealizable, it gives an upper bound on the detection performance of any practical detector implementation.

A GLRT is an easy way to implement a practical detector in which the unknown scene parameters $Q_{0}$ and $Q_{1}$ in (19) are replaced with their MLEs obtained from the observed data. The MLE of the covariance matrices $Q_{0}$ and $Q_{1}$ are given by the corresponding sample covariance matrices [26], i.e.,

$$
\hat{Q}_{0}=\frac{1}{M_{0}} \sum_{k=1}^{M_{0}} \underline{X}_{k_{0}} \underline{X}_{k_{0}}^{\mathrm{H}} \quad \hat{Q}_{1}=\frac{1}{M_{1}} \sum_{k=1}^{M_{1}} \underline{X}_{k_{1}} \underline{X}_{k_{1}}^{\mathrm{H}}
$$

where the pixel pairs $\underline{X}_{k_{0}}=\left[f_{k_{0}}, g_{k_{0}}\right]^{\mathrm{T}}, k=1, \ldots, M_{0}$, and $\underline{X}_{k_{1}}=\left[f_{k_{1}}, g_{k_{1}}\right]^{\mathrm{T}}, k=1, \ldots, M_{1}$, are realizations of the $H_{0}$ and $H_{1}$ hypotheses, respectively. The quality of the sample covariance estimates are dependent on obtaining a sufficient number of independent image pixels $M_{0}$ and $M_{1}$, which are characteristic of the $H_{0}$ and $H_{1}$ hypotheses associated with the local $N$-pixel area under test. In practice, this may require some use of a priori information regarding the nature of the scene changes. For example, fine-scale subtle scene changes may have little effect on the mean backscatter power, in which case it may be assumed that $\sigma_{g 0}^{2}=\sigma_{g 1}^{2}$. Alternatively, given knowledge of the terrain and sources of scene disturbance, some change in mean backscatter power may be postulated based on models of the phenomenology [29]. Estimates of the coherence $\gamma_{0}$ and interferometric phase $\Phi_{0}$ under the null hypothesis may be obtained by using sufficiently large estimation windows and/or applying masks to the imagery such that the fine-scale changes will have a negligible effect on the estimated parameters.

In the change-detection scenarios considered in this paper, such as the detection of vehicle tracks across an open field, the scene disturbances are typically localized and isolated to specific regions in the scene. Furthermore, the scene changes are also typically on a significantly smaller spatial scale than variations in the underlying statistical properties of the scene's

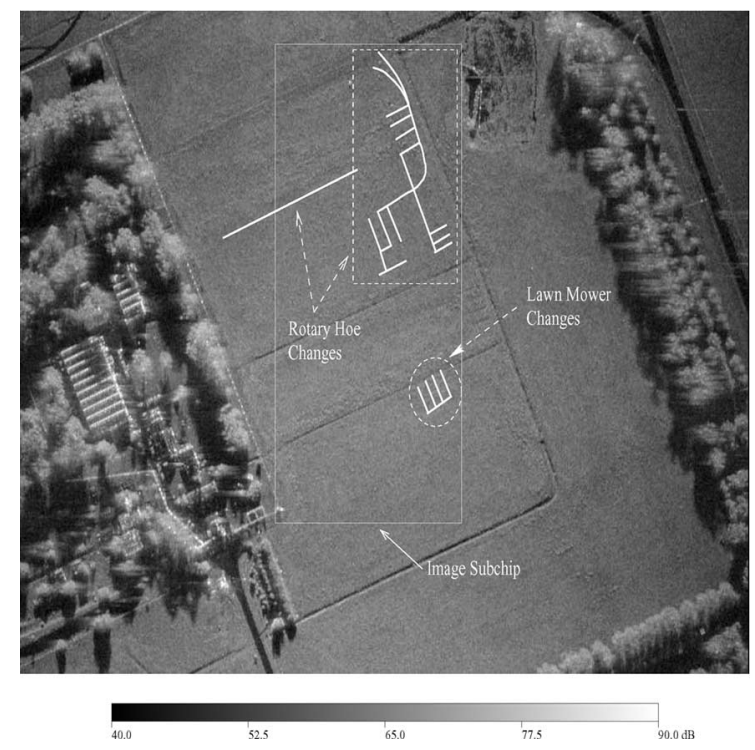

Fig. 7. Intensity SAR image of the scene acquired from the primary imaging collection. Superimposed on the image is a schematic showing the scene changes carried out with the rotary hoe and lawn mower.

complex radar backscatter. Under these conditions, large homogeneous areas for estimating the unknown covariance matrices may be readily identified. Indeed, using a fine-resolution sensor and processing a wide-angle collection aperture, estimation windows consisting of a statistically significant number of samples, suitable for estimating the unknown covariance matrices, may be readily obtained.

Closed-form expressions for the detection performance of the GRLT have not been found. However, Monte Carlo simulation techniques may be used to evaluate the average detection performance that may be expected for typical sample sizes $M_{0}$ and $M_{1}$ used in the estimation of $Q_{0}$ and $Q_{1}$, respectively. Fig. 6 shows the average ROC curves of the log-likelihood change statistic obtained via Monte Carlo simulation for sample window sizes of $M_{0}=M_{1}=25,64,121$, and 400 , and $N=7$. The change-detection scenario considered in the simulation has $\sigma_{f}^{2}=\sigma_{g 0}^{2}=\sigma_{g 1}^{2}$ and $\gamma_{0}=0.62$. From Fig. 6, it is evident that, to achieve an average detection performance comparable to that of the clairvoyant detector, window sizes of the order of several hundred pixels are required.

\section{Application to Experimental Data}

To investigate the detection performance of the proposed change statistic, a repeat-pass interferometric experiment was conducted with the DSTO Ingara X-band horizontally polarized SAR. In this experiment, repeat-pass collections of a flat lightly grassed field were obtained. The field shown in the intensity SAR image of Fig. 7 has drainage trenches approximately $1.5-\mathrm{m}$ wide and $0.8-\mathrm{m}$ deep that are visible as lines of low backscatter running through the field. The field is bordered by a line of trees on the right-hand side and by buildings and trees on the left-hand side. The field itself consisted mostly of short grass $10-15 \mathrm{~cm}$ in height with some sparse dry crop stubble in parts of the scene. The SAR image was acquired by coherently processing an azimuthal aperture of $8.5^{\circ}$ at a $16-\mathrm{km}$ standoff range and an incidence angle of $75^{\circ}$. The image has a $3-\mathrm{dB}$ resolution of $0.52 \mathrm{~m}$ in range and $0.15 \mathrm{~m}$ in azimuth, with a 
Hamming window applied, and pixel spacings of $0.35 \mathrm{~m} \times$ $0.11 \mathrm{~m}$. The intensity image in Fig. 7 has been averaged using a $2 \times 6$ pixel sliding window. The scene changes outlined in Fig. 7 consist of a series of strips of varying lengths and widths carried out with a lawn mower and a rotary hoe. The second image of the scene was acquired $2 \mathrm{~h}$ later, and the difference in grazing angle and slant range to the scene center between the two images at aperture midpoint were $0.035^{\circ}$ and $69.6 \mathrm{~m}$, respectively. For this interferometric baseline, a height change of $6.3 \mathrm{~m}$ would result in a full $2 \pi$ cycle in the interferometric phase [10].

Fig. 8(a) shows the mean backscatter-power ratio $[\hat{R}$ in (8)] map for the primary and repeat-pass image pair, while Fig. 8(b) and (c) shows the coherence and interferometric phase, respectively. The scene changes that are difficult to discern in the mean backscatter-power ratio change map appear quite clearly in the coherence map as areas of low coherence and as areas of rapid random-phase fluctuations in the interferometric phase map. Other areas of low coherence include the shadowed areas as well as the road. In these areas, there is little backscattered signal evident in the imagery, and hence, the estimated coherence is dominated by the uncorrelated radar system noise. The tree returns in the image also appear as areas of low coherence due to the movement of the leaves and branches, which occurs both during data collection and in the interval between collection.

For the purposes of examining the image statistics and formulating the changed- and unchanged-scene hypotheses, an image subchip encompassing the scene changes has been extracted from the repeat-pass image pair. As the primary and repeat-pass images are significantly oversampled, the extracted subchips have been subsampled by a factor of two in range and azimuth to give a subchip size of $500 \times 500$ pixels.

In order to compute the log-likelihood change statistic over the image subchip pair, estimates of $Q_{0}$ and $Q_{1}$ must first be obtained. Since the scene changes are difficult to discern in the sample mean backscatter-power ratio map of Fig. 8(a), it shall be assumed that $\sigma_{g 0}^{2}=\sigma_{g 1}^{2}=\sigma_{g}^{2}$. To allow for possible variation in the mean backscatter powers $\sigma_{f}^{2}$ and $\sigma_{g}^{2}$ across the repeat-pass subchip pair, each subchip has been partitioned into 25 nonoverlapping regions, each $100 \times 100$ pixels in size, over which sample estimates of the backscatter power have been computed by evaluating $I_{f}$ and $I_{g}$ in (6) with $N=10000$. A thin plate spline has been fitted to both the primary and repeat-pass sample estimates to model the spatial variation of the backscatter power across the scene. Fig. 9 shows the thin plate spline models obtained for the mean backscatter powers $\sigma_{f}^{2}$ and $\sigma_{g}^{2}$ of the primary and repeat-pass image subchips. The models indicate that the mean backscatter power across each of the image subchips varies by approximately $6 \mathrm{~dB}$. This may possibly be due to an error in the antenna-pointing accuracy, resulting in an antenna beam-pattern variation across the scene.

Ground-truth observations indicate that the field in the imaged scene is relatively homogeneous with respect to the type of vegetation, its coverage, and size. Therefore, the unchanged $H_{0}$ scene coherence $\gamma_{0}$ is expected to be constant, and a histogram of sample estimates computed over the entire image subchip pair may be obtained and compared to the theoretical density function given in (12). Fig. 10 shows the sample coherence histogram obtained using a $3 \times 3$ sliding estimation window

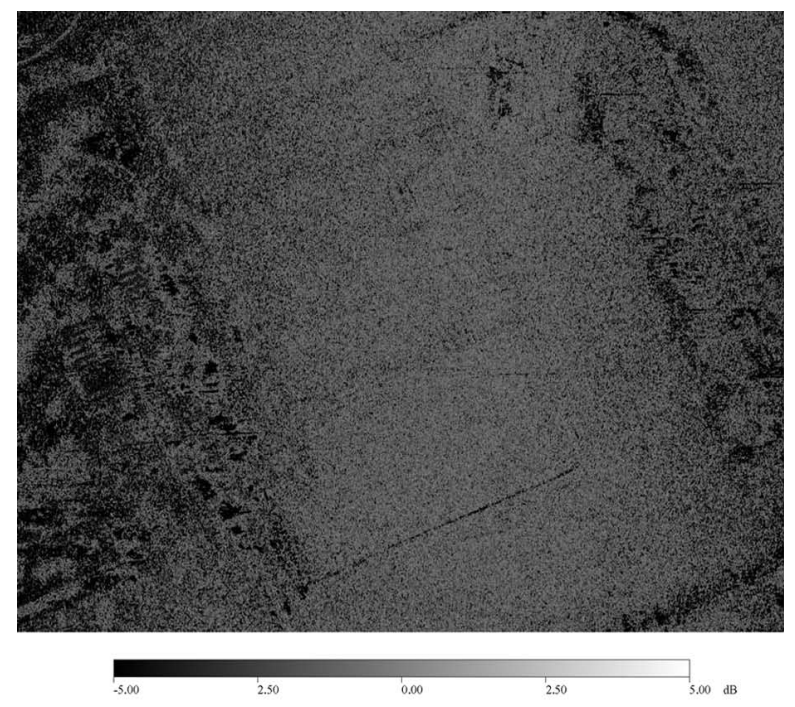

(a)

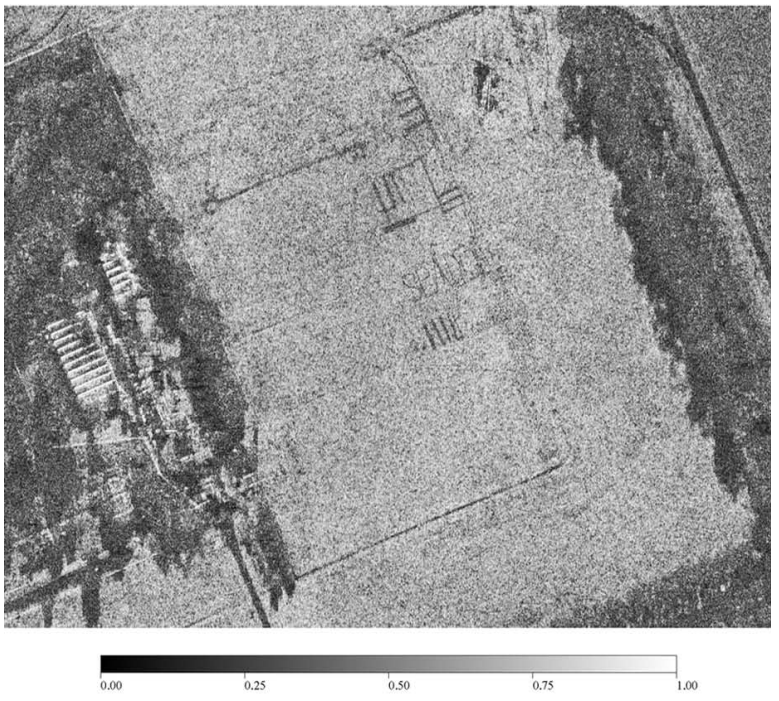

(b)

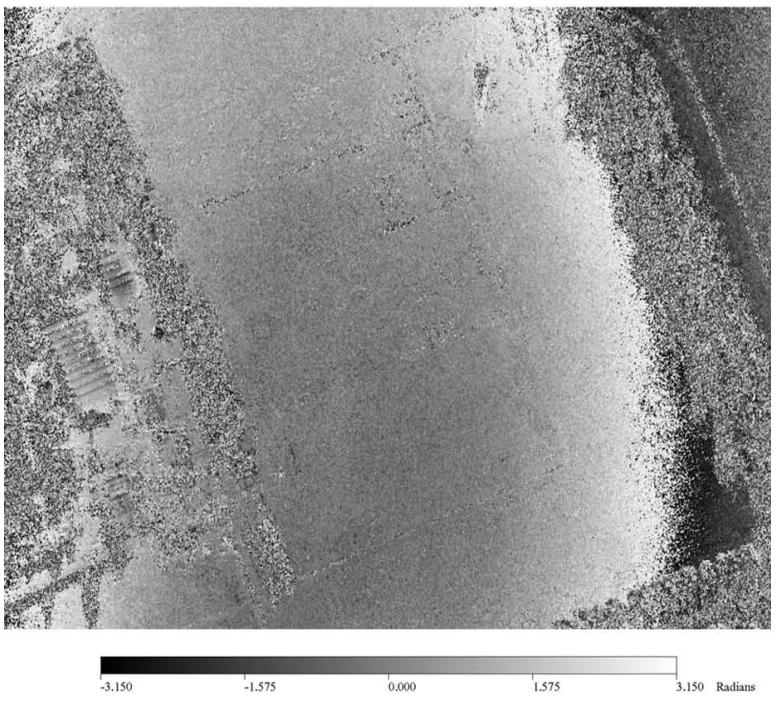

(c)

Fig. 8. Mean backscatter-power ratio, sample coherence, and interferometric phase evaluated over the repeat-pass image pair using a $2 \times 7$ pixel spatial estimation window. (a) Mean backscatter-power ratio. (b) Sample coherence. (c) Sample interferometric phase. 


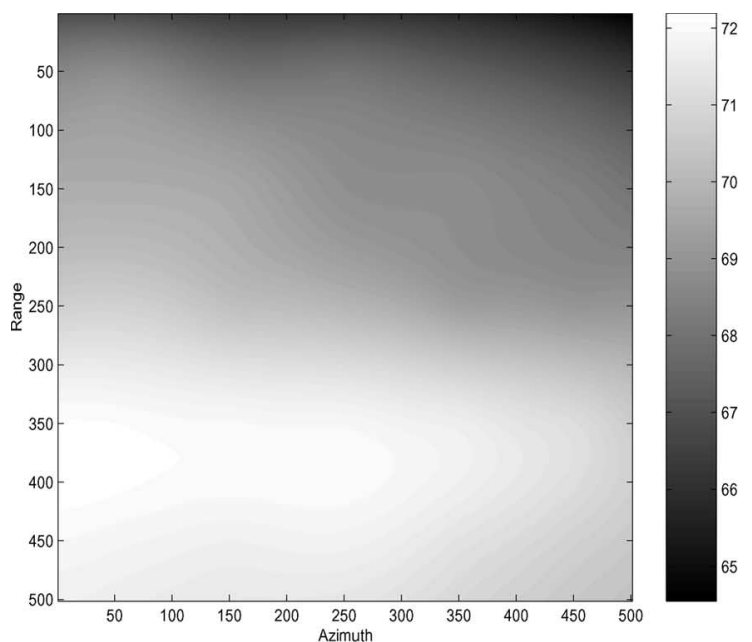

(a)

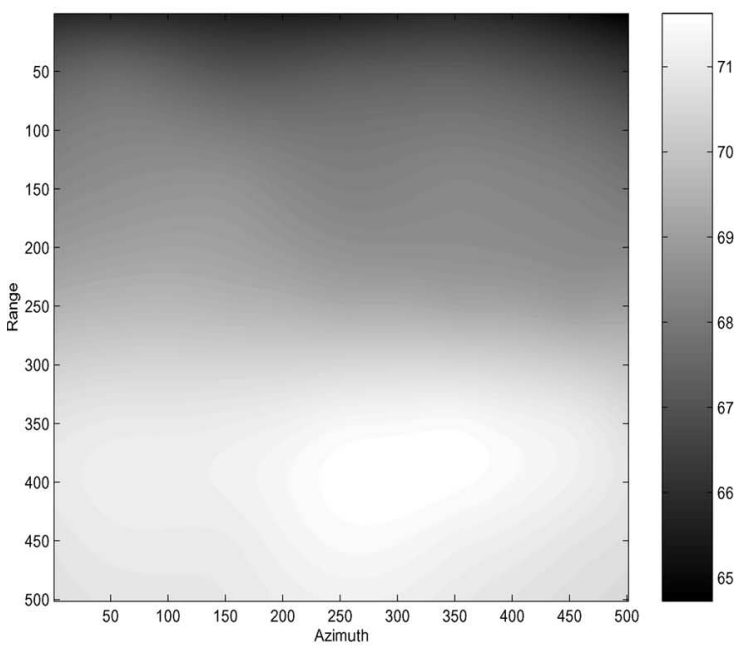

(b)

Fig. 9. Thin plate spline models describing the spatial variation of the mean backscatter power of the primary and repeat-pass image subchip pair. (a) Primary backscatter-power model (decibels). (b) Repeat-pass backscatterpower model (decibels).

applied over the entire image subchip pair. Also shown is the theoretical density function of the sample coherence where a true coherence of 0.62 and ENL of 7 have been found to provide the best fit to the experimental histogram. Note that the ENL is less than the number of pixels used in the sliding estimation window, indicating that some residual correlation due to oversampling exists between neighboring pixels in the image subchips. The theoretical density function and experimental histogram are in excellent agreement, with only a slight deviation at low-coherence values due to the inclusion of the modified low-coherence areas in the computation of the sample coherence.

The interferometric phase $\Phi_{0}$ in the unchanged-scene hypothesis may vary across the scene depending on the terrain topography and any uncompensated relative phase terms that remain after image formation. To allow for this possible spatial variation, the sample estimates of the interferometric phase obtained from the $3 \times 3$ pixel sliding estimation window have been used to generate a thin plate spline model of the interferometric-phase variation across the image subchip pair. Fig. 11 shows the sample estimates of the scene and the

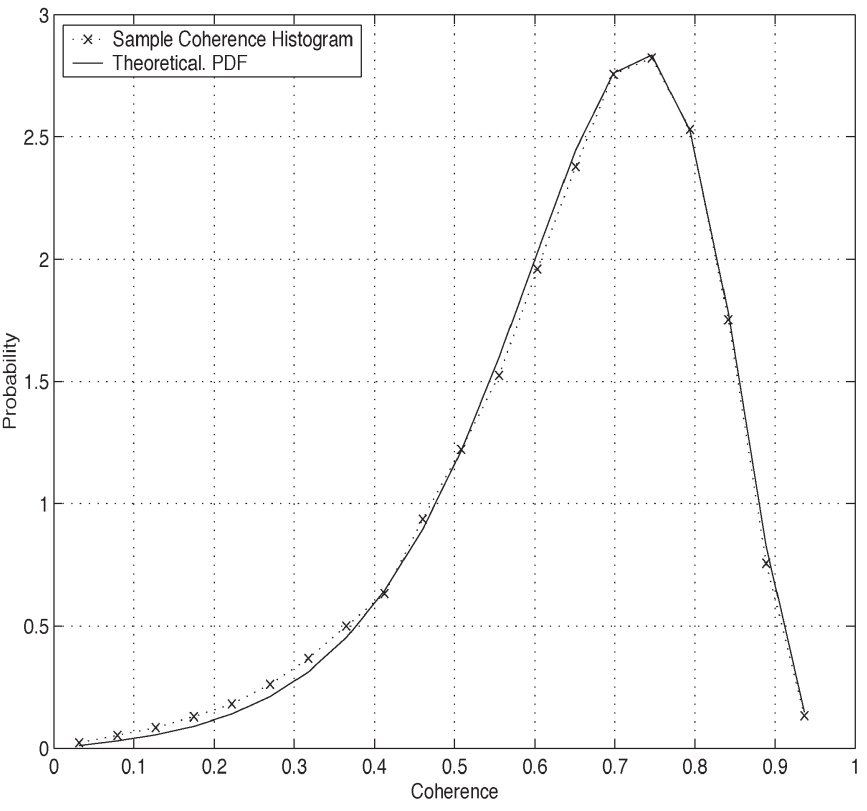

Fig. 10. Histogram and theoretical density function for the sample coherence evaluated of the primary and repeat-pass image subchips.

corresponding thin plate spline model. From the model, the phase varies by $134^{\circ}$ across the image subchip scene.

Using the models just described for the covariance matrices $Q_{0}$ and $Q_{1}$, the log-likelihood change statistic may now be computed using an $N$-pixel sliding window across the image subchip pair. Fig. 12 shows the sample coherence and loglikelihood change maps evaluated over the scene using a $3 \times 3$ pixel sliding estimation window corresponding to an ENL of 7. Also shown are the change detections obtained by applying appropriate thresholds to the coherence and log-likelihood change maps. The thresholds applied in each case have been experimentally determined to achieve a fixed number of false alarms in an area known to have no man-made ground changes, thereby giving a $P_{\mathrm{fa}}=0.018$. It is clear that the log-likelihood change statistic has a significantly better detection performance, allowing the scene disturbances to be more readily discerned. While it is difficult to quantify the experimental $P_{\mathrm{d}}$ given the size and geometry of the disturbances, an estimate based on one of the modified strips yields a $P_{\mathrm{d}}$ of 0.30 for the sample coherence and 0.68 for the log-likelihood change. These compare favorably with the theoretical values obtained from Figs. 2 and 5 of 0.31 for the sample coherence and 0.795 for the log-likelihood change statistic. The experimental probability of detection for the log-likelihood change statistic is slightly less than the theoretically predicted value. Possible reasons for this discrepancy include deviations from the theoretical density functions due to the presence of texture in the scene imagery, small errors in the estimation of the covariance matrices $Q_{0}$ and $Q_{1}$ and their spatial variation, as well as inaccuracies in the estimation of the experimental $P_{\mathrm{d}}$ due to the small sample size.

\section{CONCLUSION}

In this paper, the detection of man-made scene changes using repeat-pass SAR interferometry has been formulated as a hypothesis-testing problem. Using a bivariate Gaussian statistical description of a repeat-pass InSAR image pair, models 


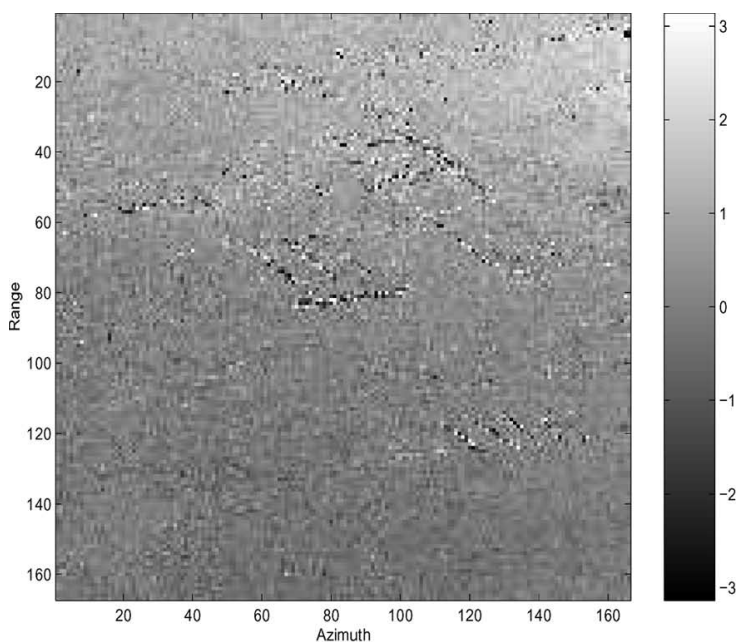

(a)

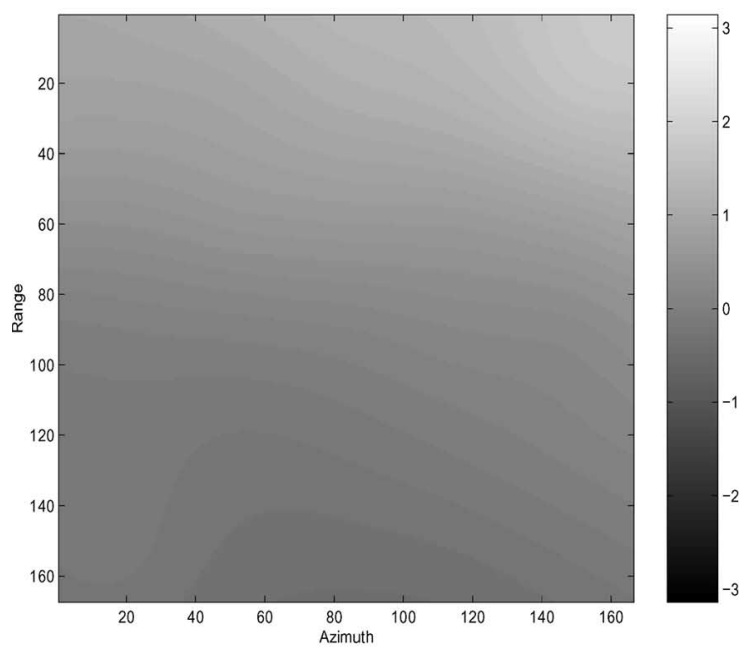

(b)

Fig. 11. Sample estimates and thin plate spline model describing the spatial variation of the interferometric phase between the primary and repeat-pass image subchip pair. (a) InSAR phase sample estimates in radians. (b) Thin plate spline model of the InSAR phase in radians.

describing the changed- and unchanged-scene hypotheses are obtained, which are parameterized by a pair of unknown covariance matrices. A log-likelihood change statistic for discriminating between the changed and unchanged hypotheses has been derived. The theoretical performance of a clairvoyant loglikelihood change statistic is quantified. The detection performance of the clairvoyant detector provides an upper bound on the performance of any practical implementation of the change statistic. It is shown that the degradation in the performance of a practical implementation of the log-likelihood change statistic is minimal, provided that sufficient sample averaging is carried out in the estimation of the unknown covariance matrices. Assuming that the scene changes of interest may be characterized by complete decorrelation, the theoretical detection performance of the log-likelihood change statistic is shown to be significantly better than that of the sample mean backscatterpower ratio and the sample coherence. The theoretical detection performance has been verified using data acquired in a repeatpass experiment conducted with the DSTO Ingara X-band SAR. The sensitivity of the change statistic to the assumptions used in formulating the scene-change and no-change models as well as
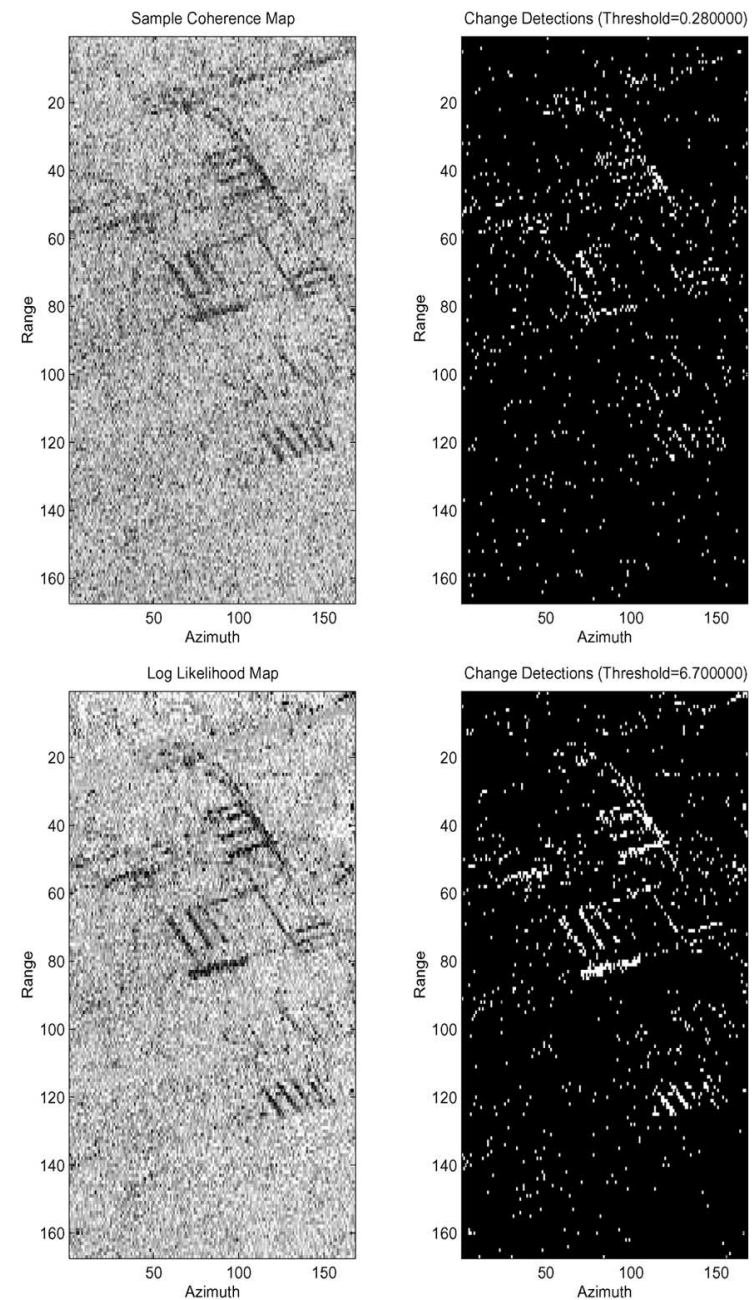

Fig. 12. Images on the left-hand side show, from top to bottom, the sample coherence and log-likelihood change-statistic maps evaluated over the subchip image pair. The images on the right-hand side show the detections obtained by applying a threshold to the corresponding change maps.

the accuracy of the parameter estimates need to be understood to determine the robustness of the approach, and is an area of future research.

\section{APPENDIX I}

The pdf of the clairvoyant log-likelihood change statistic given in (22) for the general case of $\sigma_{g 0}^{2} \neq \sigma_{g 1}^{2}$ may be derived by a direct evaluation of the integrals

$$
p(z)= \begin{cases}\int_{0}^{\infty} p(b-z, b) d b, & \text { for } z \leq 0 \\ \int_{0}^{\infty} p(a, a+z) d a, & \text { for } z>0 .\end{cases}
$$

The variables $a=\lambda_{1} \sum_{k=1}^{N}\left|u_{k}\right|^{2}$ and $b=\lambda_{2} \sum_{k=1}^{N}\left|v_{k}\right|^{2}$ are mutually dependent chi-square random variables with $2 N$ degrees of freedom, and their joint pdf $p(a, b)$ is given by (25) [28]. For $z \leq 0$, setting $a=b-z$ in (25) gives

$$
\begin{array}{r}
p(z)=\int_{0}^{\infty} \frac{\left(1-\rho^{2}\right)^{N}\left(b^{2}-z b\right)^{N-1} \exp \left\{-\left(\frac{b-z}{\alpha}+\frac{b}{\beta}\right)\right\}}{(\alpha \beta)^{N} \Gamma^{2}(N)} \\
\quad \times{ }_{1} F_{2}\left(1 ; 1, N ; \frac{\left(b^{2}-z b\right) \rho^{2}}{\alpha \beta}\right) d b .
\end{array}
$$


Substituting the series expansion for the hypergeometric function given by

$$
{ }_{1} F_{2}\left(1 ; 1, N ; \frac{a b \rho^{2}}{\alpha \beta}\right)=\sum_{k=0}^{\infty} \frac{\Gamma(N)}{\Gamma(N+k) k !}\left(\frac{a b \rho^{2}}{\alpha \beta}\right)^{k}
$$

into (51) gives

$p(z)=\frac{\left(1-\rho^{2}\right)^{N} e^{\left(\frac{z}{\alpha}\right)}}{\Gamma(N)(\alpha \beta)^{N}} \sum_{k=0}^{\infty} \mu_{k} \int_{0}^{\infty}\left(b^{2}-z b\right)^{N+k-1} \exp (-b \nu) d b$

where

$$
\begin{aligned}
\mu_{k} & =\frac{1}{\Gamma(N+k) k !}\left(\frac{\rho^{2}}{\alpha \beta}\right)^{k} \\
\nu & =\frac{1}{\alpha}+\frac{1}{\beta} .
\end{aligned}
$$

Using the binomial expansion

$\left(b^{2}-z b\right)^{N+k-1}=\sum_{p=0}^{N+k-1}\left(\begin{array}{c}N+k-1 \\ p\end{array}\right) b^{2 N+2 k-p-2}(-z)^{p}$

in (53), then

$$
\begin{aligned}
p(z)=\frac{\left(1-\rho^{2}\right)^{N} e^{\left(\frac{z}{\alpha}\right)}}{\Gamma(N)(\alpha \beta)^{N}} & \sum_{k=0}^{\infty} \mu_{k} \sum_{p=0}^{N+k-1}\left(\begin{array}{c}
N+k-1 \\
p
\end{array}\right)(-z)^{p} \\
& \times \int_{0}^{\infty} \exp (-b \nu) b^{(2 N+2 k-p-2)} d b
\end{aligned}
$$

The integral with respect to $b$ in (57) is in the form of a Gamma function [23], and thus, $p(z)$ may be written as

$$
\begin{aligned}
p(z)=\frac{\left(1-\rho^{2}\right)^{N} e^{\left(\frac{-z}{\beta}\right)}}{\Gamma(N)(\alpha \beta)^{N}} & \sum_{k=0}^{\infty}\left[\mu _ { k } \sum _ { p = 0 } ^ { N + k - 1 } \left[\left(\begin{array}{c}
N+k-1 \\
p
\end{array}\right)\right.\right. \\
& \left.\left.\times \frac{\Gamma(2 k+2 N-p-1)}{\nu^{2 k+2 N-p-1}}(z)^{p}\right]\right]
\end{aligned}
$$

where

$$
\Gamma(x)=\int_{0}^{\infty} t^{x-1} \exp (-t) d t
$$

which, for integer values of $x$, reduces to the factorial function $\Gamma(x)=(x-1)$ !. Similarly, for $z>0, p(z)$ may be written as

$$
\begin{aligned}
p(z)=\frac{\left(1-\rho^{2}\right)^{N} e^{\left(\frac{-z}{\beta}\right)}}{\Gamma(N)(\alpha \beta)^{N}} & \sum_{k=0}^{\infty}\left[\mu _ { k } \sum _ { p = 0 } ^ { N + k - 1 } \left[\left(\begin{array}{c}
N+k-1 \\
p
\end{array}\right)\right.\right. \\
& \left.\left.\times \frac{\Gamma(2 k+2 N-p-1)}{\nu^{2 k+2 N-p-1}}(z)^{p}\right]\right] .
\end{aligned}
$$

\section{APPENDIX II}

The pdf of the clairvoyant log-likelihood change statistic for the case when $\sigma_{f}^{2}=\sigma_{g 0}^{2}=\sigma_{g 1}^{2}$ may be derived by a direct evaluation of the integrals

$$
p(z)= \begin{cases}\int_{0}^{\infty} p(a) p(a+z) d a, & z>0 \\ \int_{0}^{\infty} p(b-z) p(b) d b, & z \leq 0 .\end{cases}
$$

The variables $a=\lambda_{1} \sum_{k=1}^{N}\left|u_{k}\right|^{2}$ and $b=\lambda_{2} \sum_{k=1}^{N}\left|v_{k}\right|^{2}$ are mutually independent chi-square random variables with density functions

$$
\begin{aligned}
& p(a)=\frac{1}{\Gamma(N) \lambda_{1} C_{11}}\left(\frac{a}{\lambda_{1} C_{11}}\right)^{N-1} \exp \left(-\frac{a}{\lambda_{1} C_{11}}\right) \\
& p(b)=\frac{1}{\Gamma(N) \lambda_{2} C_{22}}\left(\frac{b}{\lambda_{2} C_{22}}\right)^{N-1} \exp \left(-\frac{b}{\lambda_{2} C_{22}}\right) .
\end{aligned}
$$

For $z<0$

$$
\begin{aligned}
p(z) & =\frac{1}{\Gamma^{2}(N)\left(\lambda_{1} C_{11} \lambda_{2} C_{22}\right)^{N}} \\
& \times \int_{0}^{\infty}(b-z)^{N-1} b^{N-1} \exp \left(-\frac{b-z}{\lambda_{1} C_{11}}-\frac{b}{\lambda_{2} C_{22}}\right) d b .
\end{aligned}
$$

Using the binomial expansion

$$
(b-z)^{N-1}=\sum_{p=0}^{N-1}\left(\begin{array}{c}
N-1 \\
p
\end{array}\right) b^{N-p-1}(-z)^{p}
$$

gives

$$
\begin{aligned}
p(z)= & \frac{\exp \left(\frac{z}{\lambda_{1} C_{11}}\right)}{\Gamma^{2}(N)\left(\lambda_{1} C_{11} \lambda_{2} C_{22}\right)^{N}} \sum_{p=0}^{N-1}\left(\begin{array}{c}
N-1 \\
p
\end{array}\right)(-z)^{p} \\
& \times \int_{0}^{\infty} b^{2 N-p-2} \exp \left(-b\left(\frac{1}{\lambda_{1} C_{11}}+\frac{1}{\lambda_{2} C_{22}}\right)\right) d b .
\end{aligned}
$$

The integral in (66) is in the form of a Gamma function and so [23]

$$
\int_{0}^{\infty} b^{2 N-p-2} \exp \left(-b\left(\frac{1}{\lambda_{1} C_{11}}+\frac{1}{\lambda_{2} C_{22}}\right)\right) d b=\frac{\Gamma(2 N-p-1)}{\nu^{2 N-p-1}}
$$

where

$$
\nu=\frac{1}{\lambda_{1} C_{11}}+\frac{1}{\lambda_{2} C_{22}} .
$$

Therefore, $p(z)$ may be simplified to

$$
\begin{aligned}
p(z)=\frac{\exp \left(\frac{z}{\lambda_{1} C_{11}}\right)}{\Gamma^{2}(N)\left(\lambda_{1} C_{11} \lambda_{2} C_{22}\right)^{N}} \\
\quad \times \sum_{p=0}^{N-1}\left[\left(\begin{array}{c}
N-1 \\
p
\end{array}\right) \frac{\Gamma(2 N-p-1)}{\nu^{2 N-p-1}}(-z)^{p}\right] .
\end{aligned}
$$


Similarly, for $z>0$

$$
\begin{aligned}
p(z)=\frac{\exp \left(\frac{-z}{\lambda_{2} C_{22}}\right)}{\Gamma^{2}(N)\left(\lambda_{1} C_{11} \lambda_{2} C_{22}\right)^{N}} \\
\quad \times \sum_{p=0}^{N-1}\left[\left(\begin{array}{c}
N-1 \\
p
\end{array}\right) z^{p} \frac{\Gamma(2 N-p-1)}{\nu^{2 N-p-1}}\right] .
\end{aligned}
$$

\section{ACKNOWLEDGMENT}

The authors would like to thank the Imaging Radar Systems group for their contributions in collecting the experimental data. The authors are also grateful for the support of DSTO, The University of Adelaide, and the Cooperative Research Centre for Sensor Signal and Information Processing in facilitating this research.

\section{REFERENCES}

[1] D. Just and R. Bamler, "Phase statistics of interferograms with applications to synthetic aperture radar," Appl. Opt., vol. 33, no. 20, pp. 4361-4368, Jul. 1994.

[2] E. J. M. Rignot and J. J. van Zyl, "Change detection techniques for ERS-1 SAR data," IEEE Trans. Geosci. Remote Sens., vol. 31, no. 4, pp. 896-906, Jul. 1993.

[3] H. A. Zebker and J. Villasenor, "Decorrelation in interferometric radar echoes," IEEE Trans. Geosci. Remote Sens., vol. 30, no. 5, pp. 950-959, Sep. 1992.

[4] J. Askne, P. B. G. Dammert, L. M. H. Ulander, and G. Smith, "C-band repeat-pass interferometric SAR observations of the forest," IEEE Trans. Geosci. Remote Sens., vol. 35, no. 1, pp. 25-35, Jan. 1997.

[5] J. Askne and J. O. Hagberg, "Potential of interferometric SAR for classification of land surfaces," in Proc. Int. Geosci. Remote Sens. Symp., 1993, vol. 3, pp. 985-987.

[6] D. A. Yocky, "Interferometric SAR coherence classification utility assessment," in Proc. IEEE Int. Geosci. Remote Sens. Symp., 1998, vol. 4, pp. 1784-1786.

[7] D. Geudtner, R. Winter, and P. W. Vachon, "Flood monitoring using ERS-1 SAR interferometry coherence maps," in Proc. Int. Geosci. Remote Sens. Symp., 1996, vol. 2, pp. 966-968.

[8] D. G. Corr, "Coherence change detection for urban development monitoring," in Proc. IEE Colloq. Radar Interferometry, 1997, vol. 6, pp. 1-6.

[9] M. Preiss, "Detecting scene changes using synthetic aperture radar interferometry," Ph.D. dissertation, School Elect. Electron. Eng., Univ. Adelaide, Adelaide, Australia, 2004.

[10] C. V. Jakowatz, Jr., D. E. Wahl, P. H. Eichel, D. C. Ghiglia, and P. A. Thompson, Spotlight-Mode Synthetic Aperture Radar: A Signal Processing Approach. Boston, MA: Kluwer, 1996.

[11] S. M. Kay, Fundamentals of Statistic Signal Processing, vol. 2. Englewood Cliffs, NJ: Prentice-Hall, 1998.

[12] C. Oliver and S. Quegan, Understanding Synthetic Aperture Radar Images. Norwood, MA: Artech House, 1998.

[13] G. Franceschetti and R. Lanari, Synthetic Aperture Radar Processing. Boca Raton, FL: CRC, 1999.

[14] J. W. Goodman, Statistical Optics. Hoboken, NJ: Wiley, 1985.

[15] R. M. Goldstein, H. A. Zebker, and C. L. Werner, "Satellite radar interferometry: Two-dimensional phase unwrapping," Radio Sci., vol. 23, no. 4, pp. 713-720, Jul./Aug. 1988.

[16] H. A. Zebker and R. M. Goldstein, "Topographic mapping from interferometric SAR observations," J. Geophys. Res., vol. 91, no. B5, pp. 4993-4999, Apr. 1986.

[17] E. Rodriguez and J. M. Martin, "Theory and design of interferometric synthetic aperture radars," Proc. Inst. Elect. Eng. -F, vol. 139, no. 2, pp. 147-159, Apr. 1992.

[18] F. Gatelli, A. M. Guamieri, and F. Parizzi, "The wavenumber shift in interferometry," IEEE Trans. Geosci. Remote Sens., vol. 32, no. 4, pp. 855-865, Jul. 1994.

[19] K. P. Papathanassiou and S. R. Cloude, "Single-baseline polarimetric SAR interferometry," IEEE Trans. Geosci. Remote Sens., vol. 39, no. 11, pp. 2352-2363, Nov. 2001.
[20] M. Preiss and N. J. S. Stacy, "Scene coherency at X-band from repeat pass polarimetric interferometry," in Proc. IEEE Int. Geosci. Remote Sens. Symp., 2005, pp. 1081-1084.

[21] A. K. Gabriel, R. M. Goldstein, and H. A. Zebker, "Mapping small elevation changes over large areas: Differential radar interferometry," J. Geophys. Res., vol. 94, no. B7, pp. 9183-9191, Jul. 1989.

[22] R. Touzi, A. Lopes, and P. Bousquet, "A statistical and geometrical edge detector for SAR images," IEEE Trans. Geosci. Remote Sens., vol. 26, no. 6, pp. 764-773, Nov. 1988.

[23] M. Abramowitz and I. A. Segun, Handbook of Mathematical Functions With Formulas, Graphs and Mathematical Tables. New York: Dover, 1972.

[24] R. Touzi, A. Lopes, J. Bruniquel, and P. W. Vachon, "Coherence estimation for SAR imagery," IEEE Trans. Geosci. Remote Sens., vol. 37, no. 1, pp. 135-149, Jan. 1999.

[25] R. J. A. Tough, D. Blacknell, and S. Quegan, "A statistical description of polarimetric and interferometric synthetic aperture radar data," Proc. R. Soc. Lond. A, Math. Phys. Sci., vol. 449, no. 1937, pp. 567-589, Jun. 1995.

[26] J. W. Goodman, "Statistical analysis based on a certain multivariate complex Gaussian distribution (An introduction)," Ann. Stat. Anal., vol. 34, no. 1, pp. 152-177, Mar. 1963.

[27] H. Anton and C. Rorres, Elementary Linear Algebra With Applications. Hoboken, NJ: Wiley, 1987.

[28] J. Lee, A. R. Miller, and K. W. Hoppel, "Statistics of phase difference and product magnitude of multi-look processed Gaussian signals," Waves Random Media, vol. 4, no. 3, pp. 307-319, Jul. 1994.

[29] M. L. Williams, M. Preiss, and N. J. S. Stacy, "Performance and modelling of coherent change detection with Ingara X-band PolSAR," in Proc. 5th EUSAR Conf., 2004, pp. 829-832.

Mark Preiss (S'00-M'03) received the B.E. (Hons) and Ph.D. degrees in electrical engineering from the University of Adelaide, Adelaide, Australia, in 1994 and 2004, respectively.

From 1994 to 1996, he was with the Communications Division of the Australian Defence Science and Technology Organisation (DSTO), working on HF voiceband modem testing and design. Since 1996, he has been with the Imaging Radar Systems group at DSTO, supporting the ongoing development of the Ingara X-band synthetic aperture radar (SAR) and conducting research into imaging radar techniques. His research interests include SAR image formation, interferometric change-detection techniques, and multichannel-multibaseline interferometric SAR.

Douglas A. Gray (M'82) received the B.Sc. and Ph.D. degrees in mathematical physics from the University of Adelaide, Adelaide, Australia, in 1969 and 1974, respectively.

From 1973 to 1993, he was with the Australian Defence Science and Technology Organisation, Salisbury, applying signal processing to sonar and electronic warfare. From 1977 to 1979, he was a Visiting Scientist at the Royal Aircraft Establishment, London, U.K., and in 1985, he was a Visiting Fellow at the Australian National University, Canberra. He is currently a Professor of sensor signal processing and Deputy Directory of the Cooperative Research Centre for Sensor Signal and Information Processing at the University of Adelaide. His research interests are in the application of signal processing to sonar, radar, and electronic warfare, particularly in adaptive processes, beam forming, and signal sorting and classification techniques.

Nick J. S. Stacy (M'89) received the B.E. (Hons) degree from the University of Adelaide, Adelaide, Australia, in 1984, the M.S. degree from Stanford University, Stanford, CA, in 1985, and the Ph.D. degree from Cornell University, Ithaca, NY, in 1993, all in electrical engineering.

From 1985 to 1986, he was with the National Astronomy and Ionosphere Center at Arecibo Observatory, and from 1987 to 1989, he was with British Aerospace Australia. His work included the acquisition and analysis of Arecibo Observatory radar observations of the Moon and the analysis of Arecibo and Magellan radar data of Venus. He joined the Australian Defence Science and Technology Organisation in 1993, where he has worked in the field of imaging radar processing and analysis, primarily using the Ingara airborne radar system. He was the Australian Sensor Lead for the Global Hawk deployment to Australia in 2001 and currently leads the Imaging Radar Systems group. His research interests include signal processing and analysis of polarimetric and interferometric imaging radar. 